\title{
Sulfinamide Synthesis Using Organometallic Reagents, DABSO, and Amines
}

\author{
Pui Kin Tony Lo, Gwyndaf A. Oliver, and Michael C. Willis* \\ Cite This: J. Org. Chem. 2020, 85, 5753-5760 \\ Read Online
}

\section{ACCESS | Lل|l Metrics \& More | 回 Article Recommendations | si Supporting Information}

\begin{abstract}
We report the synthesis of sulfinamides using organometallic reagents, a sulfur dioxide reagent, and nitrogen based-nucleophiles. The addition of an organometallic reagent to the commercially available sulfur dioxide surrogate, DABSO, generates a metal sulfinate which is reacted with thionyl chloride to form a sulfinyl chloride intermediate. Trapping the sulfinyl chlorides in situ with a variety of nitrogen nucleophiles delivers sulfinamides in $32-83 \%$ yields. Each stage of the process is performed at room temperature, and the total reaction time is only $1.5 \mathrm{~h}$.
\end{abstract}

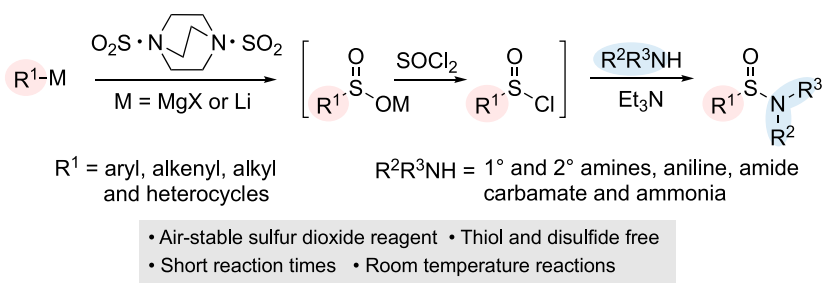

Scheme 1. Selected Methods for the Synthesis of Racemic Sulfinamides

a) From Metal Sulfinates or Sulfinic Acids organic chemistry. Chiral sulfinamides have been widely used as chiral auxiliaries, ${ }^{1}$ as ligands in transition-metal catalysis, ${ }^{2}$ and as organocatalysts. ${ }^{3}$ They can serve as $N$-protecting groups, which can be easily cleaved by acidic treatment. ${ }^{4}$ Sulfinamides are also used as intermediates to prepare sulfonimidamides and sulfoximines, which have recently enjoyed increased interest in the medicinal chemistry community. ${ }^{5}$ To date, racemic sulfinamides are usually prepared from sulfinic acids or sulfinate salts, most commonly by reaction with oxalyl chloride ${ }^{6}$ or thionyl chloride ${ }^{6 e, 7}$ to give sulfinyl chloride intermediates which then react with amines (Scheme 1a), or by direct coupling with amines using $\mathrm{DCC}^{8}$ or EDCI. ${ }^{9}$ Alternative methods include the oxidation of disulfides to form sulfinate esters ${ }^{10}$ that further react with amines ${ }^{11}$ or lithium amides (Scheme 1b). ${ }^{10 \mathrm{~b}}$ In 2007, the Harmata group reported the synthesis of sulfinamides from aryl sulfonyl chlorides and amines using $\mathrm{PPh}_{3}$ as a reductant. ${ }^{12}$ More recently, Wei and Sun reported a synthesis based on the activation of tert-butyl sulfoxides with NBS and acetic acid, subsequently quenching the reactions with a selection of nucleophiles including amines. ${ }^{13}$ Boronic acids and boronate esters have been combined with DAST-type reagents under aerobic conditions to prepare sulfinamides, as reported by the Shi laboratory (Scheme 1c). ${ }^{14}$ The oxidative copper-catalyzed synthesis of sulfinamides, starting from thiols or sulfenate anions, has also been reported, as has the copper-catalyzed trans-sulfinamidation. ${ }^{15}$ Despite the success of the methods described above, there remain limitations. For example, sulfinate salts (or their acid form), ${ }^{6-9}$ odorous thiols, ${ }^{15 a, b}$

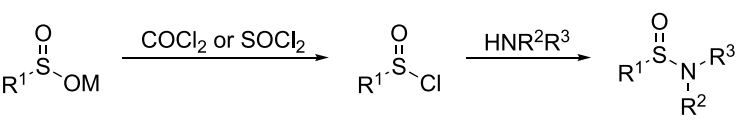

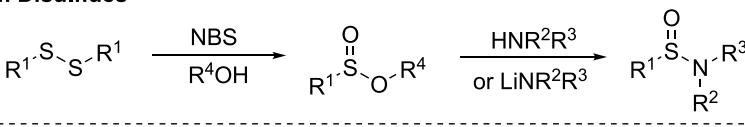

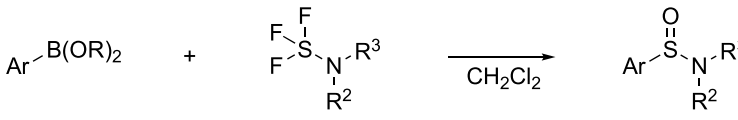$$
\text { Mulfinates or Sulfinic Acids }
$$

d) This work: From Organometallic Reagents, DABSO and Nitrogen-Based Nucleophiles

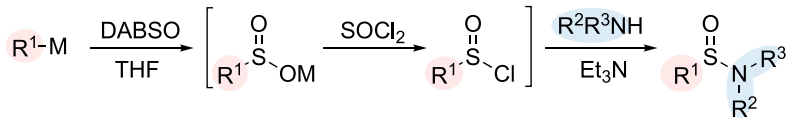

$$
\begin{aligned}
& \begin{array}{c}
\mathrm{R}^{1}=\begin{array}{c}
\text { aryl, alkenyl, alkyl } \\
\text { and heterocycles }
\end{array} \\
\mathrm{R}^{2} \mathrm{R}^{3} \mathrm{NH}=1^{\circ} \text { and } 2^{\circ} \text { amines, aniline, amide } \\
\text { carbamate and ammonia }
\end{array}
\end{aligned}
$$

Received: February 10, 2020

Published: April 14, 2020 
disulfides, ${ }^{10 a, 13,15 a}$ and sulfonyl chlorides ${ }^{12}$ are frequently reacted directly (Scheme 1a) or employed as precursors to the starting materials, and such preinstalled sulfur functional groups can limit commercial availability, especially in complex structural settings. Additionally, nitrogen based-nucleophiles are generally limited to secondary and primary amines. ${ }^{13-15}$ Ammonia could only be used when sulfinyl chlorides were employed as intermediates. ${ }^{1 \mathrm{~b}, 6 \mathrm{a}, \mathrm{c}, \mathrm{e}, 7 \mathrm{a}}$ Preformed amination reagents have also been used, ${ }^{14,15 b, c}$ which are not commercially available and require additional steps to prepare. The range of sulfinamides accessible is generally limited to aryl and alkyl variants, while the preparation of heteroaryl sulfinamides was less common using these methods. The exceptions to this were the reactions between sulfinyl chlorides and amines ${ }^{6 e}$ and the direct reaction of heteroaryl boronic acids and DAST-type reagents. ${ }^{14}$

Based on the limitations of the reported methods, we sought a more general sulfinamide synthesis and in particular targeted a method that could provide a range of sulfinyl core structures and also tolerate a variety of nitrogen functional groups. In 2010, our laboratory reported the $\operatorname{DABCO} \cdot\left(\mathrm{SO}_{2}\right)_{2}$ adduct, $\mathrm{DABSO}$, as an air-stable, and easy-to-handle sulfur dioxide surrogate. ${ }^{16}$ DABSO now has wide commercial availability. Addition of organometallic reagents to DABSO results in formation of the corresponding sulfinates, which have been converted in situ to a variety of useful sulfonyl functional groups, such as sulfones, sulfonamides, and sulfonyl fluorides. ${ }^{16 \mathrm{~b}, 17}$ We speculated that the metal sulfinates formed in situ could be telescoped with subsequent reactions to prepare sulfinamides, thus avoiding the use of substrates with preinstalled sulfur functionality. Such a transformation, based on the use of organometallic reagents, either obtained from commercial sources or prepared from metal-halogen exchange or direct deprotonation, should allow access to a wide range of complex sulfinamides.

The synthesis of sulfinyl chlorides from the addition of organometallic reagents to sulfur dioxide to form metal sulfinate salts, which were then treated with thionyl chloride, has been reported before; ${ }^{18 a}$ for example, the Ellman group employed this method to prepare sulfinamide $\mathbf{1}$ as a single diastereomer (Scheme 2). ${ }^{18 \mathrm{~b}}$ However, liquid sulfur dioxide

Scheme 2. Stepwise Synthesis of Sulfinamide from Grignard Reagents Using Sulfur Dioxide

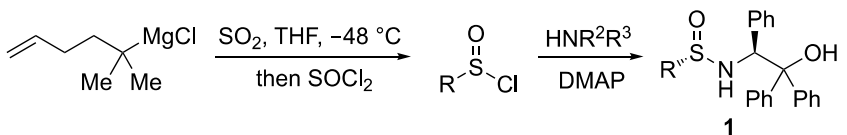

used at low temperature, obtained from condensing the gaseous reagent, was employed in this transformation and as such provides an obstacle to use in certain laboratories. We envisioned that using DABSO as a more convenient sulfur dioxide reagent would deliver a streamlined, user-friendly sulfinamide synthesis.

\section{RESULTS AND DISCUSSION}

Prior work had shown that a range of sulfinyl chlorides, including heteroaryl sulfinyl chlorides, had shown promising reactivity with nitrogen-based nucleophiles, ${ }^{6 e}$ and as such, our approach was based on reacting in situ formed metal sulfinates with either thionyl chloride or oxalyl chloride to prepare sulfinyl chloride intermediates. We were pleased to find that the in situ formed $p$-fluorophenylsulfinate salt, prepared from the addition of $p$-fluorophenylmagnesium bromide to DABSO, reacted smoothly with 1.1 equiv of thionyl chloride to form the corresponding sulfinyl chloride. This was then combined with 1.5 equiv of morpholine and 1.5 equiv of triethylamine to deliver sulfinamide $2 \mathrm{a}$ in $83 \%$ yield on a $0.5 \mathrm{mmol}$ scale and $79 \%$ yield on a gram scale (Table 1). Each step of this one-pot,

Table 1. Scope of the Organometallic Reagent ${ }^{a}$

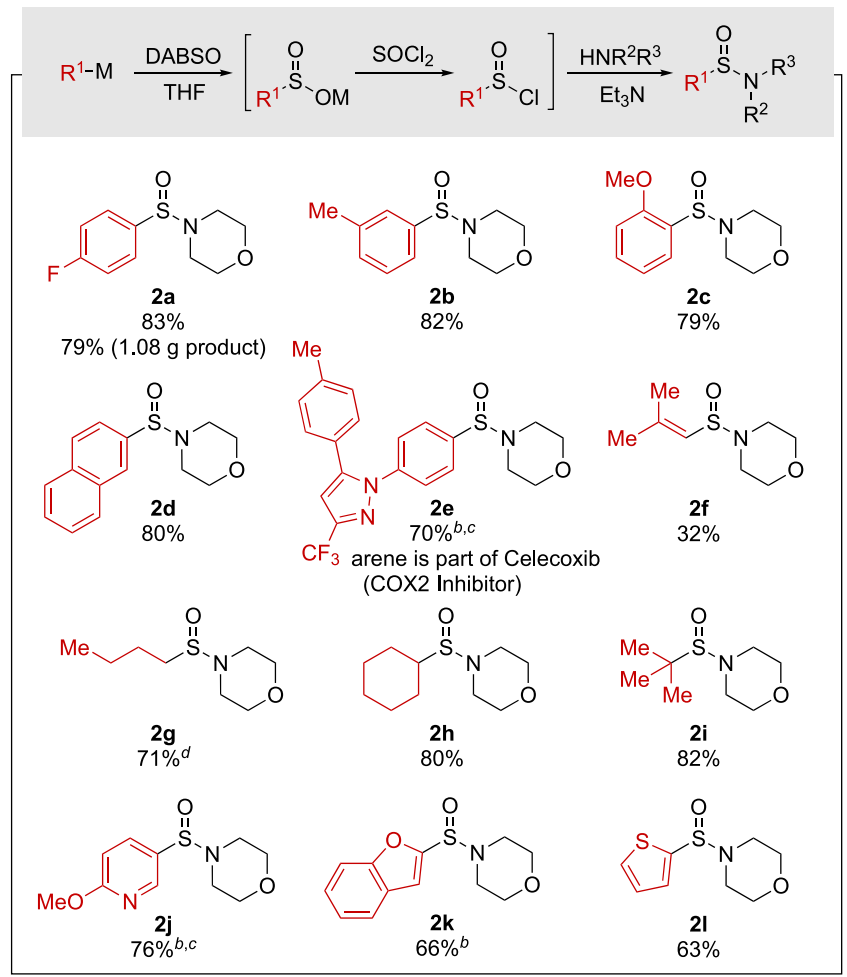

${ }^{a}$ Reaction conditions: $\mathrm{RMgX}(0.5 \mathrm{mmol}, 1$ equiv), DABSO (0.25 mmol, 0.5 equiv), THF, rt, $30 \mathrm{~min}$, then $\mathrm{SOCl}_{2}$ (1.1 equiv), rt, $30 \mathrm{~min}$ followed by $\mathrm{Et}_{3} \mathrm{~N}$ ( 1.5 equiv) and morpholine (1.5 equiv), rt, $30 \mathrm{~min}$. ${ }^{b}$ Organolithium reagent was used. ${ }^{c} \mathrm{~A}$ suspension of DABSO (0.6 equiv) in THF was added to the organolithium reagent at $-78{ }^{\circ} \mathrm{C}$ then warmed to rt. ${ }^{d_{5}} \mathrm{~min}$ for step 2 .

three-step sequence was performed at room temperature under a nitrogen atmosphere. Oxalyl chloride could be employed in place of thionyl chloride; however, the reactions with the metal sulfinates were more vigorous and needed to be performed at 0 ${ }^{\circ} \mathrm{C}$. More significantly, 1,2-diamides formed from oxalyl chloride and amines often coeluted with alkyl sulfinamides in flash column chromatography, resulting in purification difficulties. Accordingly, thionyl chloride was selected for further studies.

With the optimized conditions in hand, we next examined the scope of the reaction with respect to the organometallic reagents, using morpholine as the nucleophile, to prepare the corresponding sulfinamides (Table 1 ). Generally, a wide range of aryl, alkyl, and heteroaryl organometallic reagents could be effectively converted into sulfinyl chlorides and on to sulfinamides. Aryl Grignard reagents substituted at different positions were well tolerated, delivering para- (2a, 2e), meta(2b), and ortho-substituted phenyl (2c) and naphthyl (2d) sulfinamides in $70-83 \%$ isolated yields. A pharmaceutically relevant organolithium reagent reacted smoothly under the 
optimized conditions, delivering the arene core of the COX2 inhibitor celecoxib (2e). However, alkenyl Grignard reagent (2f) was less successful, with only $32 \%$ of the targeted sulfinamide isolated. Primary (2g), secondary (2h), and tertiary (2i) alkyl Grignard reagents were reacted effectively under the optimized conditions, with $71-82 \%$ yields of the products being isolated. It is worth noting that the primary alkyl sulfinamide $(\mathbf{2 g})$ was isolated in slightly lower yield than the corresponding secondary and tertiary alkyl systems, possibly due to the instability of the sulfinyl chloride intermediate. We were pleased to find that nitrogen-, oxygen-, and sulfur-containing heteroaromatics provided effective organometallic reagents, delivering pyridine $(2 \mathbf{j})$, benzofuran $(2 \mathbf{k})$, and thiophene $(2 \mathbf{l})$ sulfinamides in $63-76 \%$ yield.

We then explored the scope of nitrogen-based nucleophiles that could be employed, using $p$-fluorophenylmagnesium bromide as the organometallic reagent (Table 2). Secondary

\section{Table 2. Scope of Nitrogen-Based Nucleophiles ${ }^{a}$}

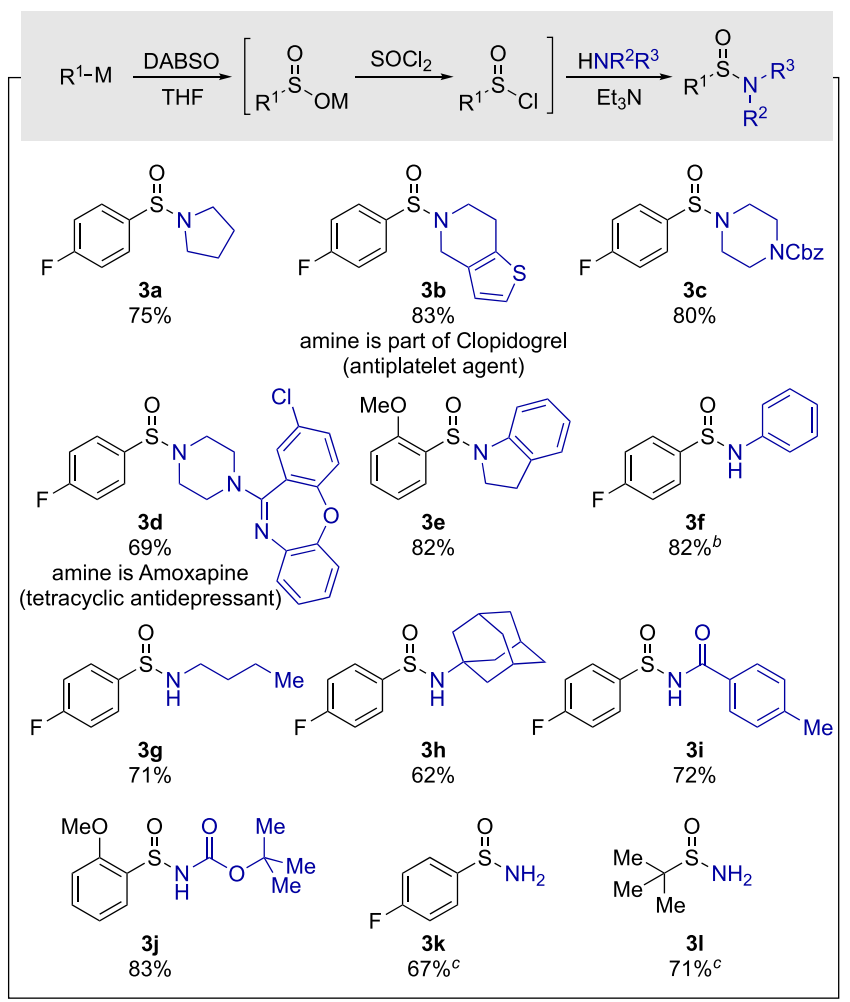

${ }^{a}$ Reaction conditions: $\mathrm{RMgX}(0.5 \mathrm{mmol}, 1$ equiv), DABSO (0.25 mmol, 0.5 equiv), THF, rt, $30 \mathrm{~min}$, then $\mathrm{SOCl}_{2}$ (1.1 equiv), rt, $30 \mathrm{~min}$ followed by $\mathrm{Et}_{3} \mathrm{~N}$ ( 1.5 equiv) and nucleophile (1.5 equiv), rt, $30 \mathrm{~min}$. Nucleophile was added as a solution in THF if it was a solid. ${ }^{b}$ Sulfinyl chloride was added to a solution of aniline (3.0 equiv) in THF. ${ }^{c}$ Sulfinyl chloride was added to a biphasic mixture of aq $\mathrm{NH}_{3}$ /ethyl acetate at $0{ }^{\circ} \mathrm{C}$.

amines, including pyrrolidine $(3 \mathbf{a})$, piperidine $(3 \mathbf{b})$, piperazine (3c, 3d), and indoline (3e), as well as biologically relevant amines (3b, 3d) reacted smoothly to provide tertiary sulfinamides in $69-83 \%$ isolated yields. Aniline (3f) was also a suitable substrate. However, for this example, a significant amount of the double $\mathrm{N}$-substituted sulfinamide was formed as a side product, indicating that the nitrogen in the initially formed sulfinamide was still available for a second nucleophilic attack on the sulfinyl chloride intermediate. We were pleased to find that this could be suppressed by transferring the formed sulfinyl chloride to a solution of aniline, allowing an $82 \%$ yield of the targeted sulfinamide to be achieved. Primary amines were also good substrates, with both $n$-butylamine $(3 \mathrm{~g})$ and sterically demanding adamantylamine $(3 \mathbf{h})$ being employed. Nucleophiles with reduced nucleophilicities, namely amide (3i) and carbamate (3j), were also effective substrates, providing $72 \%$ and $83 \%$ yields of the corresponding sulfinamides, respectively. These types of amide- and carbamate-substituted sulfinamides are typically synthesized by derivatization of the corresponding primary sulfinamides using strong base (e.g., $n-\mathrm{BuLi}$ ) in combination with anhydrides $^{6 a, 19}$ or dicarbonates (e.g., Boc anhydride), ${ }^{19 c, 20}$ and their direct synthesis from sulfinyl chlorides (or sulfinic anhydrides) is rare. ${ }^{21}$ It is worth noting that, unlike the anilinesubstituted sulfinamide, there were no significant amounts of double-substituted sulfinamides formed when primary amines $(\mathbf{3 g}, \mathbf{3 h})$, amide $(\mathbf{3 i})$, and carbamate $(3 \mathbf{j})$ nucleophiles were employed. Sulfinamides $3 \mathbf{e}$ and $3 \mathbf{j}$ feature an alternative arene core, as the corresponding $p$-fluorophenylmagnesium bromide derived products were difficult to purify using flash column chromatography. Finally, we found that a biphasic mixture of ammonium hydroxide solution and ethyl acetate proved efficient for preparing primary sulfinamides $3 \mathbf{k}$ and the racemic form of Ellman's auxiliary 31, in $67 \%$ and $71 \%$ yield, respectively. $^{6 c}$

In conclusion, we have developed a high-yielding one-pot synthesis of sulfinamides using organometallic reagents and nitrogen based-nucleophiles, exploiting sulfinyl chlorides as the reactive intermediates. The developed chemistry combines Grignard or organolithium reagents with commercially available DABSO as the source of sulfur dioxide to prepare metal sulfinates which are subsequently treated with thionyl chloride to form sulfinyl chloride intermediates. A broad range of organometallics were compatible with the process, including (hetero)aryl and alkyl systems. Secondary and primary amines, aniline, amide, carbamate, and ammonia all proved suitable $\mathrm{N}$ nucleophiles, allowing access to previously difficult to synthesize sulfinamides. The developed protocol can be performed on a preparative gram scale. Several biologically relevant aryl cores and amines were employed, providing a good demonstration of the suitability of this method for the synthesis of complex sulfinamides. Given the increasing interest in sufinamides, we anticipate wide uptake of the reported method.

\section{EXPERIMENTAL SECTION}

Reactions were performed under inert nitrogen atmosphere with anhydrous solvent unless otherwise stated. Anhydrous tetrahydrofuran (99.5\%, Extra dry over molecular sieves, stabilized, AcroSeal) was purchased. DABSO was prepared using a literature method and dried under reduced pressure $(<1 \mathrm{mbar})$ for at least $2 \mathrm{~h}$ before use. ${ }^{16 \mathrm{a}}$ Glassware was oven-dried and allowed to cool to room temperature under nitrogen. Cooling to 0 and $-78{ }^{\circ} \mathrm{C}$ was achieved using icewater and dry ice-acetone baths, respectively. Reactions were monitored by HPLC analysis and/or thin-layer chromatography (TLC) using precoated aluminum-backed silica plates (Merck Kieselgel 60 F254). Plates were visualized under ultraviolet light $(254 \mathrm{~nm})$ followed by staining with $\mathrm{KMnO}_{4}$. Flash column chromatography was carried out using Geduran Si 60, 40-63 $\mu \mathrm{m}$ silica gel; the compound to be purified was preabsorbed onto silica before loading. Petrol refers to the fraction of light petroleum ether boiling in the range $40-60{ }^{\circ} \mathrm{C} .{ }^{1} \mathrm{H},{ }^{13} \mathrm{C}\left\{{ }^{1} \mathrm{H}\right\}$, and ${ }^{19} \mathrm{~F}$ NMR spectra were recorded using 400, 101, and $376 \mathrm{MHz}$ spectrometers, 
respectively. Chemical shifts $(\delta)$ are reported in parts per million (ppm) from the residual solvent peak, and coupling constants $(J)$ are given in hertz $(\mathrm{Hz})$ and rounded to the nearest $0.5 \mathrm{~Hz}$. Lowresolution electrospray ionization (ESI) mass spectra were recorded on a Waters LCT Premier spectrometer. High-resolution mass spectra were recorded on a Brüker MicroTOF spectrometer using ESI conditions by the internal service at the Chemistry Research Laboratory, University of Oxford. Infrared spectra were recorded on a Brüker Tensor 27 FT-IR spectrometer.

General Procedure for the One-Pot Synthesis of Sulfinamides. Predried DABSO (60 mg, $0.25 \mathrm{mmol}, 0.5$ equiv) was added to an oven-dried $10 \mathrm{~mL}$ reaction vial. The vial was then sealed with a rubber septum, evacuated, and filled with $\mathrm{N}_{2}(\times 3)$. Anhydrous THF $(2 \mathrm{~mL})$ was added. The organometallic reagent $(0.50 \mathrm{mmol}, 1.0$ equiv) was added dropwise to the resulting suspension at $\mathrm{rt}$, and the reaction mixture was stirred for $30 \mathrm{~min}$. $\mathrm{SOCl}_{2}(40 \mu \mathrm{L}, 0.55 \mathrm{mmol}, 1.1$ equiv) was then added dropwise, and the mixture was stirred at $\mathrm{rt}$ for $30 \mathrm{~min}$. After this, $\mathrm{Et}_{3} \mathrm{~N}(110 \mu \mathrm{L}, 0.75 \mathrm{mmol}, 1.5$ equiv $)$ was added followed by the corresponding amine $(0.75 \mathrm{mmol}, 1.5$ equiv $)$. The mixture was stirred at $\mathrm{rt}$ for $30 \mathrm{~min}$, quenched with brine $(10 \mathrm{~mL})$, and extracted with EtOAc $(3 \times 10 \mathrm{~mL})$. A few drops of water were added to dissolve any solid formed during the workup. The combined organic phases were dried $\left(\mathrm{Na}_{2} \mathrm{SO}_{4}\right)$, filtered, and concentrated. Purification by flash column chromatography afforded the product.

4-((4-Fluorophenyl)sulfinyl)morpholine (2a). Prepared according to the general procedure using 4-fluorophenylmagnesium bromide ( $0.55 \mathrm{~mL}, 0.91 \mathrm{M}$ in THF, $0.50 \mathrm{mmol}, 1.0$ equiv) and morpholine (70 $\mu \mathrm{L}, 0.75 \mathrm{mmol}, 1.5$ equiv). Purification by flash column chromatography (50\% EtOAc in petrol) afforded the title product as a white solid (95 mg, 83\%). Gram scale synthesis of $2 \mathrm{a}$ : the same procedure was followed using 4-fluorophenylmagnesium bromide $(6.0 \mathrm{~mL}, 1.01$ $\mathrm{M}$ in THF, $6.0 \mathrm{mmol}, 1.0$ equiv), DABSO $(721 \mathrm{mg}, 3.0 \mathrm{mmol}, 0.5$ equiv), $\mathrm{SOCl}_{2}$ (0.48 mL, $6.6 \mathrm{mmol}, 1.1$ equiv), $\mathrm{Et}_{3} \mathrm{~N}$ ( $1.25 \mathrm{~mL}, 9.0$ mmol, 1.5 equiv), morpholine $(0.79 \mathrm{~mL}, 9.0 \mathrm{mmol}, 1.5$ equiv), and THF $(24 \mathrm{~mL})$, affording the title product $(1.08 \mathrm{~g}, 79 \%): R_{f}(50 \%$ EtOAc in petrol $)=0.32 ;{ }^{1} \mathrm{H} \mathrm{NMR}\left(400 \mathrm{MHz}, \mathrm{CDCl}_{3}\right) \delta 7.65-7.57$ $(\mathrm{m}, 2 \mathrm{H}), 7.20-7.11(\mathrm{~m}, 2 \mathrm{H}), 3.71-3.61(\mathrm{~m}, 4 \mathrm{H}), 3.11(\mathrm{ddd}, J=$ 12.0, 6.0, $3.5 \mathrm{~Hz}, 2 \mathrm{H}), 2.90$ (ddd, $J=12.0,6.0,3.5 \mathrm{~Hz}, 2 \mathrm{H}) ;{ }^{13} \mathrm{C}\left\{{ }^{1} \mathrm{H}\right\}$ $\operatorname{NMR}\left(101 \mathrm{MHz}, \mathrm{CDCl}_{3}\right) \delta 164.5\left(\mathrm{~d},{ }^{1} J_{\mathrm{CF}}=251.5 \mathrm{~Hz}\right), 138.0\left(\mathrm{~d},{ }^{4} J_{\mathrm{CF}}\right.$ $=3.0 \mathrm{~Hz}), 128.5\left(\mathrm{~d},{ }^{3} J_{\mathrm{CF}}=9.0 \mathrm{~Hz}\right), 116.2\left(\mathrm{~d},{ }^{2} J_{\mathrm{CF}}=22.5 \mathrm{~Hz}\right), 66.9$, 45.8; ${ }^{19} \mathrm{~F}$ NMR (376 MHz, $\left.\mathrm{CDCl}_{3}\right) \delta-109.0$; LRMS $\left(\mathrm{ESI}^{+}\right) \mathrm{m} / z$ $481.7\left([2 \mathrm{M}+\mathrm{Na}]^{+}\right)$. Data is consistent with the literature. ${ }^{14}$

4-(m-Tolylsulfinyl)morpholine (2b). Prepared according to the general procedure using $m$-tolylmagnesium chloride $(0.65 \mathrm{~mL}, 0.77 \mathrm{M}$ in THF, $0.50 \mathrm{mmol}, 1.0$ equiv) and morpholine $(70 \mu \mathrm{L}, 0.75 \mathrm{mmol}$, 1.5 equiv). Purification by flash column chromatography (50\% EtOAc in petrol) afforded the title product as a colorless oil (92 $\mathrm{mg}, 82 \%): R_{f}$ $(50 \%$ EtOAc in petrol $)=0.27 ;{ }^{1} \mathrm{H} \mathrm{NMR}\left(400 \mathrm{MHz}, \mathrm{CDCl}_{3}\right) 7.48(\mathrm{~s}$, $1 \mathrm{H}), 7.43(\mathrm{~d}, J=8.0 \mathrm{~Hz}, 1 \mathrm{H}), 7.38($ app t $J=7.5 \mathrm{~Hz}, 1 \mathrm{H}), 7.28(\mathrm{~d}, J$ $=7.0 \mathrm{~Hz}, 1 \mathrm{H}), 3.77-3.63(\mathrm{~m}, 4 \mathrm{H}), 3.19-3.10(\mathrm{~m}, 2 \mathrm{H}), 3.01-2.91$ (m, 2H), $2.42(\mathrm{~s}, 3 \mathrm{H}) ;{ }^{13} \mathrm{C}\left\{{ }^{1} \mathrm{H}\right\}$ NMR $\left(101 \mathrm{MHz}, \mathrm{CDCl}_{3}\right) \delta 142.1$, 139.1, 131.9, 128.8, 126.5, 123.2, 66.9, 45.8, 21.4; LRMS $\left(\mathrm{ESI}^{+}\right) \mathrm{m} / z$ $226.0\left([\mathrm{M}+\mathrm{H}]^{+}\right), 248.0\left([\mathrm{M}+\mathrm{Na}]^{+}\right)$. Data is consistent with the literature. $^{15 \mathrm{~b}}$

4-((2-Methoxyphenyl)sulfinyl)morpholine (2c). Prepared according to the general procedure using 2-methoxyphenylmagnesium bromide solution ( $0.49 \mathrm{~mL}, 1.02 \mathrm{M}$ in THF, $0.50 \mathrm{mmol}, 1.0$ equiv) and morpholine ( $70 \mu \mathrm{L}, 0.75 \mathrm{mmol}, 1.5$ equiv). Purification by flash column chromatography (60\% EtOAc in petrol) afforded the title product as a pale yellow oil which solidified on standing to an offwhite solid (95 mg, 79\%): $R_{f}\left(60 \%\right.$ EtOAc in petrol) $=0.27 ;{ }^{1} \mathrm{H} \mathrm{NMR}$ $\left(400 \mathrm{MHz}, \mathrm{CDCl}_{3}\right) \delta 7.81(\mathrm{~d}, J=7.5 \mathrm{~Hz}, 1 \mathrm{H}), 7.45($ app t, $J=8.0 \mathrm{~Hz}$, $1 \mathrm{H}), 7.12($ app t, $J=7.5 \mathrm{~Hz}, 1 \mathrm{H}), 6.94(\mathrm{~d}, J=8.0 \mathrm{~Hz}, 1 \mathrm{H}), 3.88(\mathrm{~s}$, $3 \mathrm{H}), 3.72-3.61(\mathrm{~m}, 4 \mathrm{H}), 3.16-3.08(\mathrm{~m}, 2 \mathrm{H}), 2.94-2.86(\mathrm{~m}, 2 \mathrm{H})$; ${ }^{13} \mathrm{C}\left\{{ }^{1} \mathrm{H}\right\} \mathrm{NMR}\left(101 \mathrm{MHz}, \mathrm{CDCl}_{3}\right) \delta 156.9,132.9,129.6,127.2$, 120.9, 111.4, 67.3, 56.0, 45.4; LRMS $\left(\mathrm{ESI}^{+}\right) \mathrm{m} / z 264.0\left([\mathrm{M}+\mathrm{Na}]^{+}\right)$. Data is consistent with the literature. ${ }^{14}$

4-(Naphthalen-2-ylsulfinyl)morpholine (2d). Prepared according to the general procedure using 2-naphthylmagnesium bromide $(0.98$ $\mathrm{mL}, 0.51 \mathrm{M}$ in THF, $0.50 \mathrm{mmol}, 1.0$ equiv) and morpholine $(70 \mu \mathrm{L}$,
$0.75 \mathrm{mmol}, 1.5$ equiv). Purification by flash column chromatography (50\% EtOAc in petrol) afforded the title product as a pale yellow viscous oil (105 mg, 80\%): $R_{f}(50 \%$ EtOAc in petrol $)=0.23 ;{ }^{1} \mathrm{H}$ NMR (400 MHz, $\left.\mathrm{CDCl}_{3}\right) \delta 8.27(\mathrm{~s}, 1 \mathrm{H}), 7.99-7.85(\mathrm{~m}, 3 \mathrm{H}), 7.63-$ $7.52(\mathrm{~m}, 3 \mathrm{H}), 3.78-3.63(\mathrm{~m}, 4 \mathrm{H}), 3.20(\mathrm{ddd}, J=12.0,6.0,3.5 \mathrm{~Hz}$, $2 \mathrm{H}), 2.98$ (ddd, $J=12.0,6.0,3.5 \mathrm{~Hz}, 2 \mathrm{H}) ;{ }^{13} \mathrm{C}\left\{{ }^{1} \mathrm{H}\right\} \mathrm{NMR}(101 \mathrm{MHz}$, $\left.\mathrm{CDCl}_{3}\right) \delta 139.5,134.5,132.8,129.1,128.9,128.03,127.96,127.3$, 122.0, 67.0, 46.0, $1 \times \operatorname{ArC}$ not observed; LRMS $\left(\mathrm{ESI}^{+}\right) \mathrm{m} / z 284.0$ $\left([\mathrm{M}+\mathrm{Na}]^{+}\right)$. Data is consistent with the literature. ${ }^{15 \mathrm{~b}}$

4-((4-(5-(p-Tolyl)-3-(trifluoromethyl)-1H-pyrazol-1-yl)phenyl)sulfinyl)morpholine (2e). 1-(4-Bromophenyl)-5-( $p$-tolyl)-3-(trifluoromethyl)-1H-pyrazole ${ }^{22}$ (191 mg, $0.50 \mathrm{mmol}, 1.0$ equiv) and anhydrous THF $(5 \mathrm{~mL})$ were added to an oven-dried $25 \mathrm{~mL} \mathrm{RBF}$ under $\mathrm{N}_{2}$ (balloon) and cooled to $-78{ }^{\circ} \mathrm{C}$. $n$-Butyllithium $(0.21 \mathrm{~mL}$, $2.37 \mathrm{M}$ in THF, $0.50 \mathrm{mmol}, 1.0$ equiv) was added dropwise, and the reaction was stirred at $-78{ }^{\circ} \mathrm{C}$ for $1 \mathrm{~h}$. A sonicated suspension (prepared in an oven-dried vial under $\mathrm{N}_{2}$ ) of predried DABSO (72 $\mathrm{mg}, 0.30 \mathrm{mmol}, 0.6$ equiv) in anhydrous THF $(5 \mathrm{~mL})$ was added slowly before warming to rt for $30 \mathrm{~min}$. $\mathrm{SOCl}_{2}(40 \mu \mathrm{L}, 0.55 \mathrm{mmol}, 1.1$ equiv) was then added dropwise, and the mixture was stirred at $\mathrm{rt}$ for $30 \mathrm{~min}$. $\mathrm{Et}_{3} \mathrm{~N}(110 \mu \mathrm{L}, 0.75 \mathrm{mmol}, 1.5$ equiv) and morpholine $(70 \mu \mathrm{L}$, $0.75 \mathrm{mmol}, 1.5$ equiv) were added. The mixture was stirred at $\mathrm{rt}$ for $30 \mathrm{~min}$, quenched with brine $(10 \mathrm{~mL})$, and extracted with EtOAc (3 $\times 10 \mathrm{~mL}$ ). A few drops of water were added to dissolve any solid formed during the workup. The combined organic phases were dried $\left(\mathrm{MgSO}_{4}\right)$, filtered, and concentrated. Purification by flash column chromatography (50\% EtOAc in petrol) afforded the title product as a pale yellow viscous oil $(154 \mathrm{mg}, 70 \%): R_{f}(50 \%$ EtOAc in petrol $)=$ $0.31 ;{ }^{1} \mathrm{H}$ NMR $\left(400 \mathrm{MHz}, \mathrm{CDCl}_{3}\right) \delta 7.69-7.63(\mathrm{~m}, 2 \mathrm{H}), 7.50-7.44$ $(\mathrm{m}, 2 \mathrm{H}), 7.16-7.11(\mathrm{~m}, 2 \mathrm{H}), 7.11-7.06(\mathrm{~m}, 2 \mathrm{H}), 6.73(\mathrm{~s}, 1 \mathrm{H})$, $3.77-3.62(\mathrm{~m}, 4 \mathrm{H}), 3.20-3.11(\mathrm{~m}, 2 \mathrm{H}), 2.99-2.89(\mathrm{~m}, 2 \mathrm{H}), 2.35(\mathrm{~s}$, $3 \mathrm{H}) ;{ }^{13} \mathrm{C}\left\{{ }^{1} \mathrm{H}\right\}$ NMR $\left(101 \mathrm{MHz}, \mathrm{CDCl}_{3}\right) \delta 145.3,143.9\left(\mathrm{q},{ }^{2} J_{\mathrm{CF}}=\right.$ $38.5 \mathrm{~Hz}), 142.4,141.6,139.7,129.7,128.8,127.3,125.9,125.8,121.2$ $\left(\mathrm{q},{ }^{1} \mathrm{~J}_{\mathrm{CF}}=269.5 \mathrm{~Hz}\right), 106.0,66.9,46.0,21.4 ;{ }^{19} \mathrm{~F}$ NMR $(377 \mathrm{MHz}$, $\left.\mathrm{CDCl}_{3}\right) \delta$-62.4; LRMS $\left(\mathrm{ESI}^{+}\right) \mathrm{m} / z$ 436.0 $\left([\mathrm{M}+\mathrm{H}]^{+}\right) ; 458.0([\mathrm{M}+$ $\mathrm{Na}]^{+}$); HRMS (ESI) $m / z[\mathrm{M}+\mathrm{H}]^{+}$calcd for $\mathrm{C}_{21} \mathrm{H}_{21} \mathrm{O}_{2} \mathrm{~N}_{3} \mathrm{~F}_{3} \mathrm{~S}$ 436.1301, found 436.1296; IR (thin film, $\nu_{\max } / \mathrm{cm}^{-1}$ ) 1472, 1373, 1235, 1160, 1133, 1096, 1069, 976, 916, 807, 729.

4-((2-Methylprop-1-en-1-yl)sulfinyl)morpholine (2f). Prepared according to the general procedure using 2-methyl-1-propenylmagnesium bromide ( $1.01 \mathrm{~mL}, 0.49 \mathrm{M}$ in THF, $0.50 \mathrm{mmol}, 1.0$ equiv), THF ( $5 \mathrm{~mL}$ ), and morpholine ( $70 \mu \mathrm{L}, 0.75 \mathrm{mmol}, 1.5$ equiv). Purification by flash column chromatography $(0-5 \% \mathrm{MeOH}$ in EtOAc) afforded the title product as a pale yellow oil $(30 \mathrm{mg}, 32 \%): R_{f}(100 \%$ EtOAc) $=0.23 ;{ }^{1} \mathrm{H} \mathrm{NMR}\left(400 \mathrm{MHz}, \mathrm{CDCl}_{3}\right) \delta 5.93$ (app. h, $\left.J=1.5 \mathrm{~Hz}, 1 \mathrm{H}\right)$, $3.74($ app t $J=5.0 \mathrm{~Hz}, 4 \mathrm{H}), 3.11($ app dd, $J=5.5,4.0 \mathrm{~Hz}, 4 \mathrm{H}), 1.91$ $(\mathrm{d}, J=1.0 \mathrm{~Hz}, 3 \mathrm{H}), 1.87(\mathrm{~d}, J=1.0 \mathrm{~Hz}, 3 \mathrm{H}) ;{ }^{13} \mathrm{C}\left\{{ }^{1} \mathrm{H}\right\} \operatorname{NMR}(101$ $\left.\mathrm{MHz} \mathrm{CDCl}_{3}\right) \delta 147.9,129.1,66.9,45.4,25.8,20.1$; LRMS $\left(\mathrm{ESI}^{+}\right) \mathrm{m} /$ $z 190.0\left([\mathrm{M}+\mathrm{H}]^{+}\right) ; 212.0\left([\mathrm{M}+\mathrm{Na}]^{+}\right)$; HRMS (ESI) $m / z[\mathrm{M}+$ $\mathrm{H}]^{+}$calcd for $\mathrm{C}_{8} \mathrm{H}_{16} \mathrm{O}_{2} \mathrm{NS}$ 190.0896, found 190.0896; IR (thin film, $\left.\nu_{\max } / \mathrm{cm}^{-1}\right) 2855,1635,1442,1258,1110,1064,1037,914,795,695$.

4-(Butylsulfinyl)morpholine (2g). Prepared according to the general procedure using butylmagnesium chloride $(0.23 \mathrm{~mL}, 2.14$ $\mathrm{M}$ in THF, $0.50 \mathrm{mmol}, 1.0$ equiv) and morpholine $(70 \mu \mathrm{L}, 0.75$ mmol, 1.5 equiv). The step 2 was left for 5 min instead. Purification by flash column chromatography (100\% EtOAc) afforded the title product as a pale yellow oil $(68 \mathrm{mg}, 71 \%): R_{f}(100 \%$ EtOAc $)=0.24$; ${ }^{1} \mathrm{H}$ NMR (400 MHz, CDCl $\left.{ }_{3}\right) \delta 3.82-3.69(\mathrm{~m}, 4 \mathrm{H}), 3.21-3.06(\mathrm{~m}$, $4 \mathrm{H}), 2.83-2.67(\mathrm{~m}, 2 \mathrm{H}), 1.58(\mathrm{app} \mathrm{dq}, J=15.0,8.0 \mathrm{~Hz}, 2 \mathrm{H}), 1.44(\mathrm{p}$, $J=7.5 \mathrm{~Hz}, 2 \mathrm{H}), 0.93(\mathrm{t}, J=7.5 \mathrm{~Hz}, 3 \mathrm{H}) ;{ }^{13} \mathrm{C}\left\{{ }^{1} \mathrm{H}\right\} \mathrm{NMR}(101 \mathrm{MHz}$, $\left.\mathrm{CDCl}_{3}\right) \delta 67.0,51.9,45.9,25.6,22.1,13.8$; LRMS $\left(\mathrm{ESI}^{+}\right) \mathrm{m} / z 192.0$ $\left([\mathrm{M}+\mathrm{H}]^{+}\right), 214.0\left([\mathrm{M}+\mathrm{Na}]^{+}\right)$; HRMS (ESI) $m / z[\mathrm{M}+\mathrm{H}]^{+}$calcd for $\mathrm{C}_{8} \mathrm{H}_{18} \mathrm{O}_{2} \mathrm{NS}$ 192.1053, found 192.1054; IR (thin film, $\nu_{\max } / \mathrm{cm}^{-1}$ ) 2980, 1653, 1455, 1382, 1259, 1111, 1068, 1038, 920.

4-(Cyclohexylsulfinyl)morpholine (2h). Prepared according to the general procedure using cyclohexylmagnesium chloride $(0.26 \mathrm{~mL}$, $1.90 \mathrm{M}$ in $\mathrm{Et}_{2} \mathrm{O}, 0.50 \mathrm{mmol}, 1.0$ equiv) and morpholine $(70 \mu \mathrm{L}, 0.75$ mmol, 1.5 equiv). Purification by flash column chromatography (0$5 \% \mathrm{MeOH}$ in EtOAc) afforded the title product as a colorless oil (87 mg, 80\%): $R_{f}(5 \% \mathrm{MeOH}$ in EtOAc $)=0.50 ;{ }^{1} \mathrm{H}$ NMR $(400 \mathrm{MHz}$, 
$\left.\mathrm{CDCl}_{3}\right) \delta 3.82-3.68(\mathrm{~m}, 4 \mathrm{H}), 3.21-3.04(\mathrm{~m}, 4 \mathrm{H}), 2.75-2.63(\mathrm{~m}$, $1 \mathrm{H}), 2.15-2.05(\mathrm{~m}, 1 \mathrm{H}), 1.93-1.60(\mathrm{~m}, 4 \mathrm{H}), 1.46-1.14(\mathrm{~m}, 5 \mathrm{H})$; ${ }^{13} \mathrm{C}\left\{{ }^{1} \mathrm{H}\right\}$ NMR (101 MHz, $\left.\mathrm{CDCl}_{3}\right) \delta 67.0,58.8,46.3,27.2,26.8,25.6$, 25.3; LRMS $\left(\mathrm{ESI}^{+}\right) \mathrm{m} / z 218.2\left([\mathrm{M}+\mathrm{H}]^{+}\right), 240.0\left([\mathrm{M}+\mathrm{Na}]^{+}\right)$; HRMS (ESI) $m / z[\mathrm{M}+\mathrm{H}]^{+}$calcd for $\mathrm{C}_{10} \mathrm{H}_{20} \mathrm{O}_{2} \mathrm{NS} 218.1209$, found 218.1211; IR (thin film, $\nu_{\max } / \mathrm{cm}^{-1}$ ) 2925, 2853, 1450, 1257, 1111, 1066, 917.

4-(tert-Butylsulfinyl)morpholine (2i). Prepared according to the general procedure using tert-butylmagnesium chloride solution $(0.53$ $\mathrm{mL}, 0.94 \mathrm{M}$ in THF, $0.50 \mathrm{mmol}, 1.0$ equiv) and morpholine $(70 \mu \mathrm{L}$, $0.75 \mathrm{mmol}, 1.5$ equiv). Purification by flash column chromatography (100\% EtOAc) afforded the title product as a white solid $(79 \mathrm{mg}$, $82 \%): R_{f}(100 \% \mathrm{EtOAc})=0.35 ;{ }^{1} \mathrm{H}$ NMR $\left(400 \mathrm{MHz}, \mathrm{CDCl}_{3}\right) \delta$ 3.77-3.69 (m, 4H), 3.21-3.13 (m, 2H), 3.13-3.05 (m, 2H), $1.19(\mathrm{~s}$, $9 \mathrm{H}) ;{ }^{13} \mathrm{C}\left\{{ }^{1} \mathrm{H}\right\}$ NMR $\left(101 \mathrm{MHz}, \mathrm{CDCl}_{3}\right) \delta 67.3,58.8,47.5,23.2$; LRMS $\left(\mathrm{ESI}^{+}\right) \mathrm{m} / z 192.3\left([\mathrm{M}+\mathrm{H}]^{+}\right), 214.3\left([\mathrm{M}+\mathrm{Na}]^{+}\right)$; HRMS (ESI) $m / z[\mathrm{M}+\mathrm{H}]^{+}$calcd for $\mathrm{C}_{8} \mathrm{H}_{18} \mathrm{O}_{2} \mathrm{NS}$ 192.1053, found 192.1055. Data is consistent with enantiomerically pure compound. ${ }^{23}$

4-((6-Methoxypyridin-3-yl)sulfinyl)morpholine (2j). 5-Bromo-2methoxypyridine (65 $\mu \mathrm{L}, 0.50 \mathrm{mmol}, 1.0$ equiv) and anhydrous THF $\left(5 \mathrm{~mL}\right.$ ) were added to an oven-dried $25 \mathrm{~mL} \mathrm{RBF}$ under $\mathrm{N}_{2}$ (balloon) and cooled to $-78{ }^{\circ} \mathrm{C}$. $n$-Butyllithium $(0.21 \mathrm{~mL}, 2.37 \mathrm{M}$ in THF, $0.50 \mathrm{mmol}, 1.0$ equiv) was added dropwise and the reaction was stirred at $-78{ }^{\circ} \mathrm{C}$ for $40 \mathrm{~min}$. A sonicated suspension (prepared in an oven-dried vial under $\mathrm{N}_{2}$ ) of predried DABSO $(72 \mathrm{mg}, 0.30 \mathrm{mmol}$, 0.6 equiv) in anhydrous THF $(5 \mathrm{~mL})$ was added slowly before warming to rt for $30 \mathrm{~min}$. $\mathrm{SOCl}_{2}$ ( $40 \mu \mathrm{L}, 0.55 \mathrm{mmol}, 1.1$ equiv) was then added dropwise, and the mixture was stirred at $\mathrm{rt}$ for $30 \mathrm{~min}$. $\mathrm{Et}_{3} \mathrm{~N}(110 \mu \mathrm{L}, 0.75 \mathrm{mmol}, 1.5$ equiv) and morpholine $(70 \mu \mathrm{L}, 0.75$ mmol, 1.5 equiv) were added. The mixture was stirred at $\mathrm{rt}$ for 30 min, quenched with brine $(10 \mathrm{~mL})$, and extracted with EtOAc $(3 \times$ $10 \mathrm{~mL}$ ). A few drops of water were added to dissolve any solid formed during the workup. The combined organic phases were dried $\left(\mathrm{MgSO}_{4}\right)$, filtered and concentrated. Purification by flash column chromatography (70\% EtOAc in petrol) afforded the title product as a pale yellow viscous oil $(92 \mathrm{mg}, 76 \%): R_{f}(70 \%$ EtOAc in petrol $)=$ 0.26 ; ${ }^{1} \mathrm{H} \mathrm{NMR}\left(400 \mathrm{MHz}, \mathrm{CDCl}_{3}\right) \delta 8.35(\mathrm{dd}, J=2.5,0.5 \mathrm{~Hz}, 1 \mathrm{H})$, $7.76(\mathrm{dd}, J=8.5,2.5 \mathrm{~Hz}, 1 \mathrm{H}), 6.81$ (dd, $J=8.5,0.5 \mathrm{~Hz}, 1 \mathrm{H}), 3.95$ (s, $3 \mathrm{H})$, $3.75-3.64(\mathrm{~m}, 4 \mathrm{H}), 3.18-3.10(\mathrm{~m}, 2 \mathrm{H}), 3.02-2.93(\mathrm{~m}, 2 \mathrm{H})$; ${ }^{13} \mathrm{C}\left\{{ }^{1} \mathrm{H}\right\}$ NMR $\left(101 \mathrm{MHz}, \mathrm{CDCl}_{3}\right) \delta 166.1,146.4,136.6,130.9$, 111.5, 66.9, 54.1, 45.8; LRMS $\left(\mathrm{ESI}^{+}\right) \mathrm{m} / z 243.0\left([\mathrm{M}+\mathrm{H}]^{+}\right) ; 265.0$ $\left([\mathrm{M}+\mathrm{Na}]^{+}\right)$; HRMS (ESI) $m / z[\mathrm{M}+\mathrm{H}]^{+}$calcd for $\mathrm{C}_{10} \mathrm{H}_{15} \mathrm{O}_{3} \mathrm{~N}_{2} \mathrm{~S}$ 243.0798, found 243.0798; IR (thin film, $\nu_{\max } / \mathrm{cm}^{-1}$ ) 2854, 1587, 1477, 1366, 1281, 1257, 1095, 1069, 1015, 916, 834, 694.

4-(Benzofuran-2-ylsulfinyl)morpholine (2k). Benzofuran (55 $\mu \mathrm{L}$, $0.50 \mathrm{mmol}, 1.0$ equiv) and anhydrous THF $(1.5 \mathrm{~mL})$ were added to an oven-dried $10 \mathrm{~mL}$ reaction vial under $\mathrm{N}_{2}$ (balloon) and cooled to 0 ${ }^{\circ} \mathrm{C}$. $n$-Butyllithium ( $0.21 \mathrm{~mL}, 2.37 \mathrm{M}$ in THF, $0.50 \mathrm{mmol}, 1.0$ equiv) was added dropwise, and the reaction was stirred at $0{ }^{\circ} \mathrm{C}$ for $5 \mathrm{~min}$ before reaching to $\mathrm{rt}$ for $1 \mathrm{~h}$. The mixture was transferred via a syringe and added dropwise to a stirred suspension of predried DABSO (60 $\mathrm{mg}, 0.25 \mathrm{mmol}, 0.5$ equiv) in THF $(2.0 \mathrm{~mL})$ in an oven-dried $10 \mathrm{~mL}$ reaction vial at $\mathrm{rt}$. The mixture was then stirred at $\mathrm{rt}$ for $30 \mathrm{~min}$. $\mathrm{SOCl}_{2}(40 \mu \mathrm{L}, 0.55 \mathrm{mmol}, 1.1$ equiv) was added dropwise, and the mixture was stirred at $\mathrm{rt}$ for $30 \mathrm{~min}$. $\mathrm{Et}_{3} \mathrm{~N}(110 \mu \mathrm{L}, 0.75 \mathrm{mmol}, 1.5$ equiv) and morpholine ( $70 \mu \mathrm{L}, 0.75 \mathrm{mmol}, 1.5$ equiv) were added. The mixture was stirred at $\mathrm{rt}$ for $30 \mathrm{~min}$, quenched with brine (10 $\mathrm{mL})$ and extracted with EtOAc $(3 \times 10 \mathrm{~mL})$. A few drops of water were added to dissolve any solid formed during the workup. The combined organic phases were dried $\left(\mathrm{MgSO}_{4}\right)$, filtered, and concentrated. Purification by flash column chromatography $(40 \%$ EtOAc in petrol) afforded the title product as a yellow oil $(83 \mathrm{mg}$, $66 \%): R_{f}(40 \%$ EtOAc in petrol $)=0.25 ;{ }^{1} \mathrm{H} \mathrm{NMR}(400 \mathrm{MHz}$, $\left.\mathrm{CDCl}_{3}\right) \delta 7.65(\mathrm{app} \mathrm{dt}, J=7.5,1.0 \mathrm{~Hz}, 1 \mathrm{H}), 7.53(\mathrm{app} \mathrm{dq}, J=8.5,1.0$ $\mathrm{Hz}, 1 \mathrm{H}), 7.38$ (ddd, $J=8.5,7.5,1.5 \mathrm{~Hz}, 1 \mathrm{H}), 7.34(\mathrm{~d}, J=1.0 \mathrm{~Hz}$, $1 \mathrm{H}), 7.30($ app td, $J=7.5,1.0 \mathrm{~Hz}, 1 \mathrm{H}), 3.83-3.65(\mathrm{~m}, 4 \mathrm{H}), 3.31$ (ddd, $J=12.5,6.0,4.0 \mathrm{~Hz}, 2 \mathrm{H}$ ), 3.14 (ddd, $J=12.5,5.5,3.5 \mathrm{~Hz}, 2 \mathrm{H}$ ); ${ }^{13} \mathrm{C}\left\{{ }^{1} \mathrm{H}\right\}$ NMR $\left(101 \mathrm{MHz}, \mathrm{CDCl}_{3}\right) \delta 156.9,154.4,126.9,126.5$, $123.9,122.3,112.3,112.1,67.0,46.1$; LRMS $\left(\mathrm{ESI}^{+}\right) \mathrm{m} / z 274.0([\mathrm{M}+$ $\mathrm{Na}]^{+}$). Data is consistent with the literature. ${ }^{14}$
4-(Thiophene-2-ylsulfinyl)morpholine (2l). Prepared according to the general procedure using 2-thienylmagnesium bromide solution ( $0.59 \mathrm{~mL}, 0.85 \mathrm{M}$ in THF, $0.50 \mathrm{mmol}, 1.0$ equiv) and morpholine (70 $\mu \mathrm{L}, 0.75 \mathrm{mmol}, 1.5$ equiv). Purification by flash column chromatography $(50 \%$ EtOAc in petrol) afforded the title product as an orange oil $(68 \mathrm{mg}, 63 \%): R_{f}(50 \%$ EtOAc in petrol $)=0.31 ;{ }^{1} \mathrm{H}$ NMR $(400$ $\left.\mathrm{MHz}, \mathrm{CDCl}_{3}\right) \delta 7.61(\mathrm{dd}, J=5.0,1.5 \mathrm{~Hz}, 1 \mathrm{H}), 7.41(\mathrm{dd}, J=3.5,1.5$ $\mathrm{Hz}, 1 \mathrm{H}), 7.14(\mathrm{dd}, J=5.0,3.5 \mathrm{~Hz}, 1 \mathrm{H}), 3.81-3.70(\mathrm{~m}, 4 \mathrm{H}), 3.25-$ $3.17(\mathrm{~m}, 2 \mathrm{H}), 3.16-3.08(\mathrm{~m}, 2 \mathrm{H}) ;{ }^{13} \mathrm{C}\left\{{ }^{1} \mathrm{H}\right\}$ NMR (101 MHz, $\left.\mathrm{CDCl}_{3}\right) \delta 145.3,131.8,130.6,128.1,67.1,45.9$; LRMS $\left(\mathrm{ESI}^{+}\right) \mathrm{m} / z$ $240.0\left([\mathrm{M}+\mathrm{Na}]^{+}\right)$. Data is consistent with the literature. ${ }^{15 \mathrm{c}}$

1-((4-Fluorophenyl)sulfinyl)pyrrolidine (3a). Prepared according to the general procedure using 4-fluorophenylmagnesium bromide (0.51 mL, $0.98 \mathrm{M}$ in THF, $0.50 \mathrm{mmol}, 1.0$ equiv) and pyrrolidine (63 $\mu \mathrm{L}, 0.75 \mathrm{mmol}, 1.5$ equiv). Purification by flash column chromatography (30\% EtOAc in petrol) afforded the title product as a pale yellow oil $(80 \mathrm{mg}, 75 \%): R_{f}(30 \%$ EtOAc in petrol $)=0.24 ;{ }^{1} \mathrm{H}$ NMR $\left(400 \mathrm{MHz}, \mathrm{CDCl}_{3}\right) \delta 7.69-7.62(\mathrm{~m}, 2 \mathrm{H}), 7.20-7.12(\mathrm{~m}, 2 \mathrm{H}), 3.37-$ $3.28(\mathrm{~m}, 2 \mathrm{H}), 3.03-2.92(\mathrm{~m}, 2 \mathrm{H}), 1.90-1.78(\mathrm{~m}, 4 \mathrm{H}) ;{ }^{13} \mathrm{C}\left\{{ }^{1} \mathrm{H}\right\}$ $\operatorname{NMR}\left(101 \mathrm{MHz}, \mathrm{CDCl}_{3}\right) \delta 164.2\left(\mathrm{~d},{ }^{1} J_{\mathrm{CF}}=250.5 \mathrm{~Hz}\right), 140.4\left(\mathrm{~d},{ }^{4} J_{\mathrm{CF}}\right.$ $=3.0 \mathrm{~Hz}), 128.2\left(\mathrm{~d},{ }^{3} J_{\mathrm{CF}}=9.0 \mathrm{~Hz}\right), 116.1\left(\mathrm{~d},{ }^{2} J_{\mathrm{CF}}=22.5 \mathrm{~Hz}\right), 46.1$, 26.1; ${ }^{19} \mathrm{~F}$ NMR $\left(377 \mathrm{MHz}, \mathrm{CDCl}_{3}\right) \delta-110.2$; LRMS $\left(\mathrm{ESI}^{+}\right) \mathrm{m} / \mathrm{z}$ $236.3\left([\mathrm{M}+\mathrm{Na}]^{+}\right)$; HRMS (ESI) $\mathrm{m} / z[\mathrm{M}+\mathrm{H}]^{+}$calcd for $\mathrm{C}_{10} \mathrm{H}_{13}$ ONFS 214.0696, found 214.0699; IR (thin film, $\nu_{\max } / \mathrm{cm}^{-1}$ ) 2967, 2876, 1587, 1488, 1222, 1084, 1062, 969, 836, 814.

5-((4-Fluorophenyl)sulfinyl)-4, 5,6,7-tetrahydrothieno[3,2-c]pyridine (3b). Prepared according to the general procedure using 4fluorophenylmagnesium bromide $(0.51 \mathrm{~mL}, 0.98 \mathrm{M}$ in THF, 0.50 mmol, 1.0 equiv) and 4,5,6,7-tetrahydrothieno[3,2-c]pyridine (90 $\mu \mathrm{L}$, $0.75 \mathrm{mmol}, 1.5$ equiv). Purification by flash column chromatography (20\% EtOAc in petrol) afforded the title product as a pale yellow viscous oil $(116 \mathrm{mg}, 83 \%): R_{f}(20 \%$ EtOAc in petrol $)=0.22 ;{ }^{1} \mathrm{H}$ NMR (400 MHz, $\left.\mathrm{CDCl}_{3}\right) \delta 7.72-7.65(\mathrm{~m}, 2 \mathrm{H}), 7.23-7.16(\mathrm{~m}, 2 \mathrm{H})$, $7.10(\mathrm{~d}, J=5.0 \mathrm{~Hz}, 1 \mathrm{H}), 6.68(\mathrm{~d}, J=5.0 \mathrm{~Hz}, 1 \mathrm{H}), 4.26(\mathrm{~d}, J=15.0$ $\mathrm{Hz}, 1 \mathrm{H}), 3.90(\mathrm{~d}, J=15.0 \mathrm{~Hz}, 1 \mathrm{H}), 3.61-3.52(\mathrm{~m}, 1 \mathrm{H}), 3.48-3.39$ $(\mathrm{m}, 1 \mathrm{H}), 3.04-2.93(\mathrm{~m}, 1 \mathrm{H}), 2.92-2.83(\mathrm{~m}, 1 \mathrm{H}) ;{ }^{13} \mathrm{C}\left\{{ }^{1} \mathrm{H}\right\} \mathrm{NMR}$ $\left(101 \mathrm{MHz}, \mathrm{CDCl}_{3}\right) \delta 164.5\left(\mathrm{~d},{ }^{1} J_{\mathrm{CF}}=252.0 \mathrm{~Hz}\right), 138.7\left(\mathrm{~d},{ }^{4} J_{\mathrm{CF}}=2.9\right.$ $\mathrm{Hz}), 133.0,131.8,128.7\left(\mathrm{~d},{ }^{3} \mathrm{~J}_{\mathrm{CF}}=9.5 \mathrm{~Hz}\right), 125.1,123.5,116.3(\mathrm{~d}$, $\left.{ }^{2} J_{\mathrm{CF}}=22.5 \mathrm{~Hz}\right), 45.7,43.9,26.2 ;{ }^{19} \mathrm{~F} \mathrm{NMR}\left(377 \mathrm{MHz}, \mathrm{CDCl}_{3}\right) \delta$ -109.2; LRMS $\left(\mathrm{ESI}^{+}\right) \mathrm{m} / z 282.4\left([\mathrm{M}+\mathrm{H}]^{+}\right), 304.4\left([\mathrm{M}+\mathrm{Na}]^{+}\right)$; HRMS (ESI) $m / z[\mathrm{M}+\mathrm{Na}]^{+}$calcd for $\mathrm{C}_{13} \mathrm{H}_{12} \mathrm{ONFNaS}_{2} 304.0237$, found 304.0237; IR (thin film, $\nu_{\max } / \mathrm{cm}^{-1}$ ) 2848, 1587, 1488, 1223, 1086, 1064, 906, 836, 704.

Benzyl 4-((4-Fluorophenyl)sulfinyl)piperazine-1-carboxylate (3c). Prepared according to the general procedure using 4fluorophenylmagnesium bromide $(0.51 \mathrm{~mL}, 0.98 \mathrm{M}$ in THF, 0.50 mmol, 1.0 equiv) and benzyl piperazine-1-carboxylate $(0.15 \mathrm{~mL}, 0.75$ mmol, 1.5 equiv). Purification by flash column chromatography $(40 \%$ EtOAc in petrol) afforded the title product as a pale yellow viscous oil (145 mg, 80\%): $R_{f}(40 \%$ EtOAc in petrol $)=0.23 ;{ }^{1} \mathrm{H}$ NMR $(400$ $\left.\mathrm{MHz} \mathrm{CDCl}_{3}\right) \delta 7.68-7.61(\mathrm{~m}, 2 \mathrm{H}), 7.37-7.27(\mathrm{~m}, 5 \mathrm{H}), 7.23-7.16$ $(\mathrm{m}, 2 \mathrm{H}), 5.12(\mathrm{~s}, 2 \mathrm{H}), 3.65-3.45(\mathrm{~m}, 4 \mathrm{H}), 3.18-3.08(\mathrm{~m}, 2 \mathrm{H})$, $3.02-2.90(\mathrm{~m}, 2 \mathrm{H}) ;{ }^{13} \mathrm{C}\left\{{ }^{1} \mathrm{H}\right\} \mathrm{NMR}\left(101 \mathrm{MHz}, \mathrm{CDCl}_{3}\right) \delta 164.6(\mathrm{~d}$, $\left.{ }^{1} J_{\mathrm{CF}}=252.0 \mathrm{~Hz}\right), 155.1,138.2\left(\mathrm{~d},{ }^{4} J_{\mathrm{CF}}=3.0 \mathrm{~Hz}\right), 136.5,128.6,128.5$ $\left(\mathrm{d},{ }^{3} J_{\mathrm{CF}}=9.0 \mathrm{~Hz}\right), 128.3,128.0,116.3\left(\mathrm{~d},{ }^{2} J_{\mathrm{CF}}=22.5 \mathrm{~Hz}\right), 67.5,45.8$, 44.2; $\left.{ }^{19} \mathrm{~F} \mathrm{NMR} \mathrm{(377} \mathrm{MHz,} \mathrm{CDCl}_{3}\right) \delta-108.7$; LRMS $\left(\mathrm{ESI}^{+}\right) \mathrm{m} / z$ $385.0\left([\mathrm{M}+\mathrm{Na}]^{+}\right)$; HRMS (ESI) $m / z[\mathrm{M}+\mathrm{Na}]^{+}$calcd for $\mathrm{C}_{18} \mathrm{H}_{19} \mathrm{O}_{3} \mathrm{~N}_{2} \mathrm{FNaS} 385.0993$, found 385.1000; IR (thin film, $\nu_{\max } /$ $\mathrm{cm}^{-1}$ ) 1698, 1587, 1489, 1427, 1240,1124, 1086, 1067, 914, 837, 697.

2-Chloro-11-(4-((4-fluorophenyl)sulfinyl)piperazin-1-yl)dibenzo$[b, f][1,4]$ oxazepine $(3 d)$. Prepared according to the general procedure using 4-fluorophenylmagnesium bromide $(0.51 \mathrm{~mL}, 0.98$ $\mathrm{M}$ in THF, $0.50 \mathrm{mmol}, 1.0$ equiv) and amoxapine $(235 \mathrm{mg}, 0.75$ mmol, 1.5 equiv). Purification by flash column chromatography $(20 \%$ EtOAc in petrol) afforded the title product as a pale yellow foam (158 $\mathrm{mg}, 69 \%): R_{f}(20 \%$ EtOAc in petrol $)=0.23 ;{ }^{1} \mathrm{H}$ NMR $(400 \mathrm{MHz}$, $\left.\mathrm{CDCl}_{3}\right) \delta 7.70-7.63(\mathrm{~m}, 2 \mathrm{H}), 7.34(\mathrm{dd}, J=8.5,2.5 \mathrm{~Hz}, 1 \mathrm{H}), 7.25(\mathrm{~d}$, $J=2.5 \mathrm{~Hz}, 1 \mathrm{H}), 7.22-7.15(\mathrm{~m}, 2 \mathrm{H}), 7.15-7.09(\mathrm{~m}, 2 \mathrm{H}), 7.09-7.03$ $(\mathrm{m}, 2 \mathrm{H}), 7.01-6.95(\mathrm{~m}, 1 \mathrm{H}), 3.53(\mathrm{~s}, 4 \mathrm{H}), 3.37-3.20(\mathrm{~m}, 2 \mathrm{H})$, 
3.20-2.99 (m, 2H); ${ }^{13} \mathrm{C}\left\{{ }^{1} \mathrm{H}\right\}$ NMR (101 MHz, $\left.\mathrm{CDCl}_{3}\right) \delta 164.4(\mathrm{~d}$, $\left.{ }^{1} J_{\mathrm{CF}}=251.5 \mathrm{~Hz}\right), 159.3,158.6,151.7,139.8,138.2\left(\mathrm{~d},{ }^{4} J_{\mathrm{CF}}=3.0 \mathrm{~Hz}\right)$, $132.7,130.4,128.8,128.5\left(\mathrm{~d},{ }^{3} J_{\mathrm{CF}}=9.0 \mathrm{~Hz}\right), 127.1,125.8,124.9$, 124.7, 122.7, 120.1, $116.2\left(\mathrm{~d},{ }^{2} J_{\mathrm{CF}}=22.5 \mathrm{~Hz}\right), 47.7,45.5 ;{ }^{19} \mathrm{~F} \mathrm{NMR}$ $\left(377 \mathrm{MHz}, \mathrm{CDCl}_{3}\right) \delta-108.8$; LRMS $\left(\mathrm{ESI}^{+}\right) \mathrm{m} / z 478.1\left([\mathrm{M}+\mathrm{Na}]^{+}\right)$; HRMS (ESI) $m / z[\mathrm{M}+\mathrm{H}]^{+}$calcd for $\mathrm{C}_{23} \mathrm{H}_{20} \mathrm{O}_{2} \mathrm{~N}_{3}{ }^{35} \mathrm{ClFS} 456.0943$, found 456.0939; IR (thin film, $\nu_{\max } / \mathrm{cm}^{-1}$ ) 1561, 1471, 1399, 1238, 1086, 905, 836, 725; mp $\left(\mathrm{CH}_{2} \mathrm{Cl}_{2}\right) 108-109^{\circ} \mathrm{C}$.

1-((2-Methoxyphenyl)sulfinyl)indoline (3e). Prepared according to the general procedure using 2-methoxyphenylmagnesium bromide solution ( $0.49 \mathrm{~mL}, 1.02 \mathrm{M}$ in THF, $0.50 \mathrm{mmol}, 1.0$ equiv) and indoline $(85 \mu \mathrm{L}, 0.75 \mathrm{mmol}, 1.5$ equiv). Purification by flash column chromatography (15-25\% EtOAc in petrol) afforded the title product as a brown solid $(113 \mathrm{mg}, 82 \%): R_{f}(15 \%$ EtOAc in petrol $)=0.21 ;{ }^{1} \mathrm{H}$ NMR (400 MHz, $\mathrm{CDCl}_{3}$ ) $\delta 7.94(\mathrm{dd}, J=7.5,1.5 \mathrm{~Hz}, 1 \mathrm{H}$ ), 7.47 (ddd, $J=8.0,7.5,1.5 \mathrm{~Hz}, 1 \mathrm{H}), 7.23-7.13(\mathrm{~m}, 3 \mathrm{H}), 7.11(\mathrm{~d}, J=7.5 \mathrm{~Hz}$, $1 \mathrm{H}), 6.95-6.85(\mathrm{~m}, 2 \mathrm{H}), 4.06-3.93(\mathrm{~m}, 1 \mathrm{H}), 3.76(\mathrm{~s}, 3 \mathrm{H}), 3.13-$ $2.88(\mathrm{~m}, 3 \mathrm{H}) ;{ }^{13} \mathrm{C}\left\{{ }^{1} \mathrm{H}\right\}$ NMR $\left(101 \mathrm{MHz}, \mathrm{CDCl}_{3}\right) \delta 156.6,146.6$, $132.8,130.7,130.2,127.5,126.5,125.1,121.9,120.8,111.5,111.2$, 56.0, 42.1, 28.4; LRMS $\left(\mathrm{ESI}^{+}\right) \mathrm{m} / z 296.0\left([\mathrm{M}+\mathrm{Na}]^{+}\right), 569.2([2 \mathrm{M}+$ $\mathrm{Na}]^{+}$); HRMS (ESI) $m / z[\mathrm{M}+\mathrm{H}]^{+}$calcd for $\mathrm{C}_{15} \mathrm{H}_{16} \mathrm{O}_{2} \mathrm{NS} 274.0896$, found 274.0895; IR (thin film, $\nu_{\max } / \mathrm{cm}^{-1}$ ) 1590, 1477, 1274, 1240, 1088, 1050, 1016, 931, 749; $\mathrm{mp}\left(\mathrm{CH}_{2} \mathrm{Cl}_{2}\right) 116-118{ }^{\circ} \mathrm{C}$.

4-Fluoro-N-phenylbenzenesulfinamide ${ }^{13}$ (3f). Predried DABSO ( $60 \mathrm{mg}, 0.25 \mathrm{mmol}, 0.5$ equiv) was added to an oven-dried $10 \mathrm{~mL}$ reaction vial. The vial was then sealed with a rubber septum, evacuated, and filled with $\mathrm{N}_{2}(\times 3)$. Anhydrous THF $(2 \mathrm{~mL})$ was added. 4-Fluorophenylmagnesium bromide $(0.50 \mathrm{~mL}, 1.01 \mathrm{M}$ in THF, $0.50 \mathrm{mmol}, 1.0$ equiv) was added dropwise to the resulting suspension at $\mathrm{rt}$, and the reaction mixture was stirred for $30 \mathrm{~min}$. $\mathrm{SOCl}_{2}(40 \mu \mathrm{L}, 0.55 \mathrm{mmol}, 1.1$ equiv) was then added dropwise, and the mixture was stirred at $\mathrm{rt}$ for $30 \mathrm{~min}$. The reaction mixture was transferred via a syringe and added dropwise to a solution of $\mathrm{Et}_{3} \mathrm{~N}$ (110 $\mu \mathrm{L}, 0.75 \mathrm{mmol}, 1.5$ equiv) and aniline $(140 \mu \mathrm{L}, 1.5 \mathrm{mmol}, 3$ equiv) in THF $(2 \mathrm{~mL})$ in a $25 \mathrm{~mL}$ oven-dried RBF under $\mathrm{N}_{2}$ at rt. Anhydrous THF $(2 \mathrm{~mL} \times 2)$ was then added to the reaction vial and transferred to the $25 \mathrm{~mL} \mathrm{RBF}$ to ensure a quantitative transfer of the sulfinyl chloride. After addition, the mixture was stirred at $\mathrm{rt}$ for 30 min, quenched with brine $(10 \mathrm{~mL})$, and extracted with EtOAc $(3 \times$ $10 \mathrm{~mL}$ ). A few drops of water were added to dissolve any solid formed during the workup. The combined organic phases were dried $\left(\mathrm{MgSO}_{4}\right)$, filtered, and concentrated. Purification by flash column chromatography $(20-25 \%$ EtOAc in petrol) afforded the title product as a pale yellow solid (97 mg, 82\%): $R_{f}(20 \%$ EtOAc in petrol $)=0.21$; ${ }^{1} \mathrm{H}$ NMR (400 MHz, $\left.\mathrm{CDCl}_{3}\right) \delta 7.81-7.71(\mathrm{~m}, 2 \mathrm{H}), 7.31-7.24(\mathrm{~m}$, 2H), 7.23-7.17 (m, 2H), 7.11-7.04 (m, 3H), $6.30(\mathrm{~s}, 1 \mathrm{H}) ;{ }^{13} \mathrm{C}\left\{{ }^{1} \mathrm{H}\right\}$ NMR $\left(101 \mathrm{MHz}, \mathrm{CDCl}_{3}\right) \delta 164.7\left(\mathrm{~d},{ }^{1} J_{\mathrm{CF}}=252.0 \mathrm{~Hz}\right), 140.4,140.2$ $\left(\mathrm{d},{ }^{4} J_{\mathrm{CF}}=3.0 \mathrm{~Hz}\right), 129.6,128.1\left(\mathrm{~d},{ }^{3} J_{\mathrm{CF}}=9.0 \mathrm{~Hz}\right), 124.0,119.4,116.4$ $\left(\mathrm{d},{ }^{2} J_{\mathrm{CF}}=23.0 \mathrm{~Hz}\right) ;{ }^{19} \mathrm{~F}$ NMR $\left(377 \mathrm{MHz}, \mathrm{CDCl}_{3}\right) \delta-108.4$; LRMS $\left(\mathrm{ESI}^{+}\right) \mathrm{m} / z 258.0\left([\mathrm{M}+\mathrm{Na}]^{+}\right)$; HRMS (ESI) $m / z[\mathrm{M}+\mathrm{Na}]^{+}$calcd for $\mathrm{C}_{12} \mathrm{H}_{10} \mathrm{ONFNaS} 258.0359$, found 258.0361; IR (thin film, $\nu_{\max } /$ $\mathrm{cm}^{-1}$ ) 3174, 1586, 1487, 1224, 1154, 1085, 1055, 882, 816, 745, 688; $\mathrm{mp}\left(\mathrm{CH}_{2} \mathrm{Cl}_{2}\right): 115-117^{\circ} \mathrm{C}$.

$\mathrm{N}$-Butyl-4-fluorobenzenesulfinamide (3g). Prepared according to the general procedure using 4-fluorophenylmagnesium bromide $(0.51$ $\mathrm{mL}, 0.98 \mathrm{M}$ in THF, $0.50 \mathrm{mmol}, 1.0$ equiv) and $n$-butylamine $(74 \mu \mathrm{L}$, $0.75 \mathrm{mmol}, 1.5$ equiv). Purification by flash column chromatography (30\% EtOAc in petrol) afforded the title product as a pale yellow oil (77 mg, 71\%): $R_{f}(30 \%$ EtOAc in petrol $)=0.26 ;{ }^{1} \mathrm{H}$ NMR $(400$ $\left.\mathrm{MHz}, \mathrm{CDCl}_{3}\right) \delta 7.68-7.62(\mathrm{~m}, 2 \mathrm{H}), 7.17-7.10(\mathrm{~m}, 2 \mathrm{H}), 4.28(\mathrm{t}, J=$ $6.0 \mathrm{~Hz}, 1 \mathrm{H}), 3.11-3.01(\mathrm{~m}, 1 \mathrm{H}), 2.80-2.69(\mathrm{~m}, 1 \mathrm{H}), 1.49-1.40(\mathrm{~m}$, $2 \mathrm{H}), 1.33-1.23(\mathrm{~m}, 2 \mathrm{H}), 0.83(\mathrm{t}, J=7.3 \mathrm{~Hz}, 3 \mathrm{H}) ;{ }^{13} \mathrm{C}\left\{{ }^{1} \mathrm{H}\right\} \mathrm{NMR}$ $\left(101 \mathrm{MHz}, \mathrm{CDCl}_{3}\right) \delta 164.4\left(\mathrm{~d},{ }^{1} J_{\mathrm{CF}}=250.5 \mathrm{~Hz}\right), 140.1\left(\mathrm{~d},{ }^{4} J_{\mathrm{CF}}=3.0\right.$ $\mathrm{Hz}), 128.4\left(\mathrm{~d},{ }^{3} J_{\mathrm{CF}}=9.0 \mathrm{~Hz}\right), 116.0\left(\mathrm{~d},{ }^{2} J_{\mathrm{CF}}=22.5 \mathrm{~Hz}\right), 40.8,32.6$, 20.0, 13.7; ${ }^{19} \mathrm{~F}$ NMR $\left(377 \mathrm{MHz}, \mathrm{CDCl}_{3}\right) \delta-109.8$; LRMS $\left(\mathrm{ESI}^{+}\right) \mathrm{m} /$ $z 216.0\left([\mathrm{M}+\mathrm{H}]^{+}\right), 238.1\left([\mathrm{M}+\mathrm{Na}]^{+}\right)$. Data is consistent with the literature. $^{24}$

$3 N-((3 s, 5 s, 7 s)$-Adamantan-1-yl)-4-fluorobenzenesulfinamide (3h). Prepared according to the general procedure using 4fluorophenylmagnesium bromide $(0.50 \mathrm{~mL}, 1.01 \mathrm{M}$ in THF, 0.50 mmol, 1.0 equiv) and an emulsion of 1-adamantylamine $(114 \mathrm{mg}$, $0.75 \mathrm{mmol}, 1.5$ equiv) in THF $(3 \mathrm{~mL})$. During aqueous workup, the combined organic phases were dried over $\mathrm{MgSO}_{4}$ instead. Purification by flash column chromatography (25\% EtOAc in petrol) afforded the title product as a white solid (91 mg, 62\%): $R_{f}(25 \%$ EtOAc in petrol) $=0.29 ;{ }^{1} \mathrm{H}$ NMR $\left(400 \mathrm{MHz}, \mathrm{CDCl}_{3}\right) \delta 7.73-7.63(\mathrm{~m}, 2 \mathrm{H}), 7.20-$ $7.11(\mathrm{~m}, 2 \mathrm{H}), 3.83(\mathrm{~s}, 1 \mathrm{H}), 2.19-2.10(\mathrm{~m}, 3 \mathrm{H}), 2.04-1.94(\mathrm{~m}, 3 \mathrm{H})$, 1.94-1.86 (m, 3H), 1.75-1.63 (m, 6H); ${ }^{13} \mathrm{C}\left\{{ }^{1} \mathrm{H}\right\}$ NMR $(101 \mathrm{MHz}$, $\left.\mathrm{CDCl}_{3}\right) \delta 164.3\left(\mathrm{~d},{ }^{1} J_{\mathrm{CF}}=251.0 \mathrm{~Hz}\right), 142.4\left(\mathrm{~d},{ }^{4} J_{\mathrm{CF}}=3.0 \mathrm{~Hz}\right), 128.2$ $\left(\mathrm{d},{ }^{3} J_{\mathrm{CF}}=9.0 \mathrm{~Hz}\right), 115.9\left(\mathrm{~d},{ }^{2} J_{\mathrm{CF}}=22.0 \mathrm{~Hz}\right), 54.7,44.8,36.1,29.8$; ${ }^{19} \mathrm{~F}$ NMR $\left(376 \mathrm{MHz}, \mathrm{CDCl}_{3}\right) \delta-110.2$; LRMS $\left(\mathrm{ESI}^{+}\right) \mathrm{m} / z 294.1$ $\left([\mathrm{M}+\mathrm{H}]^{+}\right)$; HRMS (ESI) $\mathrm{m} / z[\mathrm{M}+\mathrm{H}]^{+}$calcd for $\mathrm{C}_{16} \mathrm{H}_{21} \mathrm{ONFS}$ 294.1322, found 294.1326; IR (thin film, $\nu_{\max } / \mathrm{cm}^{-1}$ ) 3090, 2981, 2903, 2849, 1588, 1490, 1452, 1396, 1224, 1159, 1086, 1040, 948, 835, 744; mp $\left(\mathrm{CH}_{2} \mathrm{Cl}_{2}\right) 264-266{ }^{\circ} \mathrm{C}$.

N-((4-Fluorophenyl)sulfinyl)-4-methylbenzamide (3i). Prepared according to the general procedure using 4-fluorophenylmagnesium bromide ( $0.50 \mathrm{~mL}, 1.01 \mathrm{M}$ in THF, $0.50 \mathrm{mmol}, 1.0$ equiv) and a solution of $p$-toluamide ( $101 \mathrm{mg}, 0.75 \mathrm{mmol}, 1.5$ equiv) in THF (4.0 $\mathrm{mL}$ ). During aqueous workup, the combined organic phases were dried over $\mathrm{MgSO}_{4}$ instead. Purification by flash column chromatography (40\% EtOAc in petrol) afforded the title product as a white solid $(100 \mathrm{mg}, 72 \%): R_{f}(40 \%$ EtOAc in petrol $)=0.38 ;{ }^{1} \mathrm{H}$ NMR $\left(400 \mathrm{MHz}, \mathrm{CDCl}_{3}\right) \delta 8.95(\mathrm{~s}, 1 \mathrm{H}), 7.75-7.66(\mathrm{~m}, 4 \mathrm{H}), 7.21(\mathrm{~d}, J=$ $8.0 \mathrm{~Hz}, 2 \mathrm{H}), 7.19-7.13(\mathrm{~m}, 2 \mathrm{H}), 2.38(\mathrm{~s}, 3 \mathrm{H}) ;{ }^{13} \mathrm{C}\left\{{ }^{1} \mathrm{H}\right\}$ NMR $(101$ $\left.\mathrm{MHz}, \mathrm{CDCl}_{3}\right) \delta 167.5,164.9(\mathrm{~d}, J=252.5 \mathrm{~Hz}), 144.3,139.5(\mathrm{~d}, J=$ $3.0 \mathrm{~Hz}), 129.6,128.7,128.3,127.5(\mathrm{~d}, J=9.0 \mathrm{~Hz}), 116.8(\mathrm{~d}, J=23.0$ $\mathrm{Hz}), 21.7 ;{ }^{19} \mathrm{~F} \mathrm{NMR}\left(377 \mathrm{MHz}, \mathrm{CDCl}_{3}\right) \delta-107.1$; LRMS $\left(\mathrm{ESI}^{+}\right) \mathrm{m} /$ $z 300.0\left([\mathrm{M}+\mathrm{Na}]^{+}\right)$; HRMS (ESI) $m / z[\mathrm{M}+\mathrm{H}]^{+}$calcd for $\mathrm{C}_{14} \mathrm{H}_{13} \mathrm{O}_{2} \mathrm{NFS}$ 278.0646, found 278.0646; IR (thin film, $\nu_{\max } / \mathrm{cm}^{-1}$ ) 3181, 1670, 1587, 1491, 1420, 1395, 1231, 1090, 1060, 888, 833, 750; $\mathrm{mp}\left(\mathrm{CH}_{2} \mathrm{Cl}_{2}\right) 135-136{ }^{\circ} \mathrm{C}$.

tert-Butyl ((2-Methoxyphenyl)sulfinyl)carbamate (3j). Prepared according to the general procedure using 2-methoxyphenylmagnesium bromide solution $(0.49 \mathrm{~mL}, 1.02 \mathrm{M}$ in THF, $0.50 \mathrm{mmol}, 1.0$ equiv) and a solution of tert-butyl carbamate ( $88 \mathrm{mg}, 0.75 \mathrm{mmol}, 1.5$ equiv) in THF $(2.0 \mathrm{~mL})$. Purification by flash column chromatography (30$40 \%$ EtOAc in petrol) afforded the title product as a colorless viscous oil $(113 \mathrm{mg}, 83 \%): R_{f}(30 \%$ EtOAc in petrol $)=0.15 ;{ }^{1} \mathrm{H}$ NMR $(400$ $\left.\mathrm{MHz} \mathrm{CDCl}_{3}\right) \delta 7.84(\mathrm{dd}, J=7.5,1.5 \mathrm{~Hz}, 1 \mathrm{H}), 7.48$ (ddd, $J=8.0,7.5$, $1.5 \mathrm{~Hz}, 1 \mathrm{H}), 7.11$ (app td, $J=7.5,1.0 \mathrm{~Hz}, 1 \mathrm{H}), 6.99$ (s, 1H), 6.95 $(\mathrm{dd}, J=8.5,1.0 \mathrm{~Hz}, 1 \mathrm{H}), 3.89(\mathrm{~s}, 3 \mathrm{H}), 1.48(\mathrm{~s}, 9 \mathrm{H}) ;{ }^{13} \mathrm{C}\left\{{ }^{1} \mathrm{H}\right\} \mathrm{NMR}$ $\left(101 \mathrm{MHz}, \mathrm{CDCl}_{3}\right) \delta 156.3,152.7,133.6,131.3,126.0,121.2,111.5$, 83.1, 56.1, 28.2.; LRMS $\left(\mathrm{ESI}^{+}\right) \mathrm{m} / z$ 294.0 $\left([\mathrm{M}+\mathrm{Na}]^{+}\right)$; HRMS (ESI) $m / z[\mathrm{M}+\mathrm{H}]^{+}$calcd for $\mathrm{C}_{12} \mathrm{H}_{17} \mathrm{O}_{4} \mathrm{NNaS} 294.0771$, found 294.0770; IR (thin film, $\nu_{\max } / \mathrm{cm}^{-1}$ ) 3156, 2979, 1707, 1590, 1478, 1369, 1276, 1241, 1154, 1087, 1051, 1019, 904, 820, 755, 727.

4-Fluorobenzenesulfinamide ( $3 k$ ). Predried DABSO (60 mg, 0.25 mmol, 0.5 equiv) was added to an oven-dried $10 \mathrm{~mL}$ reaction vial. The vial was then sealed with a rubber septum, evacuated and filled with $\mathrm{N}_{2}(\times 3)$. Anhydrous THF $(2.0 \mathrm{~mL})$ was added. 4Fluorophenylmagnesium bromide $(0.50 \mathrm{~mL}, 1.01 \mathrm{M}$ in THF, 0.50 mmol, 1.0 equiv) was added dropwise to the resulting suspension at $\mathrm{rt}$, and the reaction mixture was stirred for $30 \mathrm{~min}$. $\mathrm{SOCl}_{2}(40 \mu \mathrm{L}$, $0.55 \mathrm{mmol}, 1.1$ equiv) was then added dropwise, and the mixture was stirred at $\mathrm{rt}$ for $30 \mathrm{~min}$. The reaction mixture was transferred via a syringe and added dropwise to a stirred solution of $35 \%$ aq $\mathrm{NH}_{3}(0.5$ $\mathrm{mL})$ and EtOAc $(1.0 \mathrm{~mL})$ in a $25 \mathrm{~mL} \mathrm{RBF}$ at $0{ }^{\circ} \mathrm{C}$. Anhydrous THF $(2.0 \mathrm{~mL} \times 2)$ was added to the reaction vial and transferred to the 25 $\mathrm{mL} \mathrm{RBF}$ to ensure a quantitative transfer of the sulfinyl chloride. After addition, the mixture was stirred at $\mathrm{rt}$ for $30 \mathrm{~min}$, quenched with brine $(10 \mathrm{~mL})$, and extracted with EtOAc $(3 \times 10 \mathrm{~mL})$. A few drops of water were added to dissolve any solid formed during the workup. The combined organic phases were dried $\left(\mathrm{MgSO}_{4}\right)$, filtered, and concentrated. Purification by flash column chromatography $(100 \%$ EtOAc) afforded the title product as a white solid (53 mg, 67\%): $R_{f}$ $(100 \%$ EtOAc $)=0.50 ;{ }^{1} \mathrm{H}$ NMR $\left(400 \mathrm{MHz}, \mathrm{DMSO}-d_{6}\right) \delta 7.75-7.64$ $(\mathrm{m}, 2 \mathrm{H}), 7.41-7.30(\mathrm{~m}, 2 \mathrm{H}), 6.29(\mathrm{~s}, 2 \mathrm{H}) ;{ }^{13} \mathrm{C}\left\{{ }^{1} \mathrm{H}\right\}$ NMR $(101$ $\left.\mathrm{MHz}, \mathrm{DMSO}-d_{6}\right) \delta 163.2\left(\mathrm{~d},{ }^{1} J_{\mathrm{CF}}=247.0 \mathrm{~Hz}\right), 144.2\left(\mathrm{~d},{ }^{4} J_{\mathrm{CF}}=2.5\right.$ $\mathrm{Hz}), 127.9\left(\mathrm{~d},{ }^{3} J_{\mathrm{CF}}=9.0 \mathrm{~Hz}\right), 115.6\left(\mathrm{~d},{ }^{2} J_{\mathrm{CF}}=22.0 \mathrm{~Hz}\right) ;{ }^{19} \mathrm{~F} \mathrm{NMR}$ 
(377 MHz, DMSO) $\delta-111.4$; LRMS $\left(\mathrm{ESI}^{+}\right) \mathrm{m} / z$ 341.4 $([2 \mathrm{M}+$ $\left.\mathrm{Na}]^{+}\right)$. Data is consistent with the literature. ${ }^{19 b}$

2-Methylpropane-2-sulfinamide (3l). Predried DABSO (60 mg, $0.25 \mathrm{mmol}, 0.5$ equiv) was added to an oven-dried $10 \mathrm{~mL}$ reaction vial. The vial was then sealed with a rubber septum, evacuated, and filled with $\mathrm{N}_{2}(\times 3)$. Anhydrous THF $(2.0 \mathrm{~mL})$ was added. tertButylmagnesium chloride $(0.53 \mathrm{~mL}, 0.94 \mathrm{M}$ in THF, $0.50 \mathrm{mmol}, 1.0$ equiv) was added dropwise to the resulting suspension at $\mathrm{rt}$, and the reaction mixture was stirred for $30 \mathrm{~min}$. $\mathrm{SOCl}_{2}(40 \mu \mathrm{L}, 0.55 \mathrm{mmol}, 1.1$ equiv) was then added dropwise, and the mixture was stirred at $\mathrm{rt}$ for $30 \mathrm{~min}$. The reaction mixture was transferred via a syringe and added dropwise to a stirred solution of $35 \%$ aq $\mathrm{NH}_{3}(0.5 \mathrm{~mL})$ and EtOAc $(0.5 \mathrm{~mL})$ in a $25 \mathrm{~mL} \mathrm{RBF}$ at $0{ }^{\circ} \mathrm{C}$. Anhydrous THF $(2.0 \mathrm{~mL} \times 2)$ was added to the reaction vial and transferred to the $25 \mathrm{~mL} \mathrm{RBF}$ to ensure a quantitative transfer of the sulfinyl chloride. After addition, the mixture was stirred at $\mathrm{rt}$ for $30 \mathrm{~min}$, quenched with brine $(10 \mathrm{~mL})$, and extracted with EtOAc $(3 \times 10 \mathrm{~mL})$. A few drops of water were added to dissolve any solid formed during the workup. The combined organic phases were dried $\left(\mathrm{MgSO}_{4}\right)$, filtered, and concentrated. Purification by flash column chromatography $(0-5 \% \mathrm{MeOH}$ in EtOAc) afforded the title product as a pale yellow viscous oil $(43 \mathrm{mg}$, $71 \%): R_{f}(100 \%$ EtOAc $)=0.17 ;{ }^{1} \mathrm{H}$ NMR $\left(400 \mathrm{MHz} \mathrm{CDCl}_{3}\right) \delta 3.72$ (s, 2H), $1.22(\mathrm{~s}, 9 \mathrm{H}) ;{ }^{13} \mathrm{C}\left\{{ }^{1} \mathrm{H}\right\}$ NMR $\left(101 \mathrm{MHz}, \mathrm{CDCl}_{3}\right) \delta 55.5$, 22.3; LRMS $\left(\mathrm{ESI}^{+}\right) \mathrm{m} / z 122.0\left([\mathrm{M}+\mathrm{H}]^{+}\right) ; 144.0\left([\mathrm{M}+\mathrm{Na}]^{+}\right)$. Data is consistent with the literature. ${ }^{25}$

\section{ASSOCIATED CONTENT}

\section{(s) Supporting Information}

hThe Supporting Information is available free of charge at https://pubs.acs.org/doi/10.1021/acs.joc.0c00334.

${ }^{1} \mathrm{H},{ }^{13} \mathrm{C}$, and ${ }^{19} \mathrm{~F}$ NMR spectra of synthesized compounds (PDF)

\section{AUTHOR INFORMATION}

\section{Corresponding Author}

Michael C. Willis - Department of Chemistry, Chemistry

Research Laboratory, University of Oxford, Oxford OX1 3TA,

United Kingdom; 10 orcid.org/0000-0002-0636-6471;

Email: michael.willis@chem.ox.ac.uk

\section{Authors}

Pui Kin Tony Lo - Department of Chemistry, Chemistry Research Laboratory, University of Oxford, Oxford OX1 3TA, United Kingdom

Gwyndaf A. Oliver - Department of Chemistry, Chemistry Research Laboratory, University of Oxford, Oxford OX1 3TA, United Kingdom

Complete contact information is available at: https://pubs.acs.org/10.1021/acs.joc.0c00334

\section{Notes}

The authors declare no competing financial interest.

\section{ACKNOWLEDGMENTS}

We thank the EPSRC (EP/K024205/1) for support of this study. We thank Tony Lo for the cover design.

\section{REFERENCES}

(1) (a) Ellman, J. A.; Owens, T. D.; Tang, T. P. N-tert-Butanesulfinyl Imines: Versatile Intermediates for the Asymmetric Synthesis of Amines. Acc. Chem. Res. 2002, 35, 984-995. (b) Robak, M. T.; Herbage, M. A.; Ellman, J. A. Synthesis and Applications of tertButanesulfinamide. Chem. Rev. 2010, 110, 3600-3740.

(2) Otocka, S.; Kwiatkowska, M.; Madalińska, L.; Kiełbasiński, P. Chiral Organosulfur Ligands/Catalysts with a Stereogenic Sulfur
Atom: Applications in Asymmetric Synthesis. Chem. Rev. 2017, 117, 4147-4181.

(3) Dinér, P.; Sadhukhan, A.; Blomkvist, B. Chiral Sulfinamides as Highly Enantioselective Organocatalysts. ChemCatChem 2014, 6, 3063-3066.

(4) (a) Tang, T. P.; Volkman, S. K.; Ellman, J. A. Asymmetric Synthesis of Protected 1,2-Amino Alcohols Using tert-Butanesulfinyl Aldimines and Ketimines. J. Org. Chem. 2001, 66, 8772-8778. (b) Fritz, S. P.; Mumtaz, A.; Yar, M.; McGarrigle, E. M.; Aggarwal, V. K. Sulfinamides as Highly Effective Amine Protecting Groups and Their Use in the Conversion of Amino Alcohols into Morpholines. Eur. J. Org. Chem. 2011, 2011, 3156-3164.

(5) (a) Nandi, G. C.; Arvidsson, P. I. Sulfonimidamides: Synthesis and Applications in Preparative Organic Chemistry. Adv. Synth. Catal. 2018, 360, 2976-3001. (b) Lucking, U. Sulfoximines: A Neglected Opportunity in Medicinal Chemistry. Angew. Chem., Int. Ed. 2013, 52, 9399-9408. (c) Mendonca Matos, P.; Lewis, W.; Moore, J. C.; Stockman, R. A. Sulfonimidates: Useful Synthetic Intermediates for Sulfoximine Synthesis via C-S Bond Formation. Org. Lett. 2018, 20, 3674-3677.

(6) (a) Backes, B. J.; Dragoli, D. R.; Ellman, J. A. Chiral N-Acyl-tertbutanesulfinamides: The "Safety-Catch" Principle Applied to Diastereoselective Enolate Alkylations. J. Org. Chem. 1999, 64, 5472-5478. (b) Coulomb, J.; Certal, V.; Fensterbank, L.; Lacôte, E.; Malacria, M. Formation of Cyclic Sulfinates and Sulfinamides through Homolytic Substitution at the Sulfur Atom. Angew. Chem., Int. Ed. 2006, 45, 633-637. (c) Savile, C. K.; Kazlauskas, R. J. The 3(3-Pyridine)propionyl Anchor Group for Protease-Catalyzed Resolutions: $p$-Toluenesulfinamide and Sterically Hindered Secondary Alcohols. Adv. Synth. Catal. 2006, 348, 1183-1192. (d) Zhu, R.-H.; Shi, X.-X. Practical and Highly Stereoselective Method for the Preparation of Several Chiral Arylsulfinamides and Arylsulfinates Based on the Spontaneous Crystallization of Diastereomerically Pure $N$-Benzyl-N-(1-Phenylethyl)-Arylsulfinamides. Tetrahedron: Asymmetry 2011, 22, 387-393. (e) Miller, D.; Thom, S.; St-Gallay, S.; Shannon, J.; Leeson, P. Novel Compounds. WO2019068772 (A1), 2019.

(7) (a) Kawęcki, R. Facile Synthesis of Homochiral Derivatives of 10-Bornane Sulfinates, Sulfinamides and Sulfinimines. Tetrahedron: Asymmetry 1999, 10, 4183-4190. (b) Li, X.-B.; Xu, Z.-F.; Liu, L.-J.; Liu, J.-T. Synthesis and Identification of Solution-Stable Sulfenic Acids: Perfluoroalkanesulfenic Acids. Eur. J. Org. Chem. 2014, 2014, $1182-1188$

(8) Furukawa, M.; Okawara, T. Convenient Syntheses of Sulfinamide Derivatives. Synthesis 1976, 1976, 339-340.

(9) Gafur, S. H.; Waggoner, S. L.; Jacobsen, E.; Hamaker, C. G.; Hitchcock, S. R. Efficient Synthesis of Sulfinate Esters and Sulfinamides via Activated Esters of $p$-Toluenesulfinic Acid. Synthesis 2018, 50, 4855-4866.

(10) (a) Brownbridge, P.; Jowett, I. C. 'One-Pot' Synthesis of Sulphinic Esters from Disulphides. Synthesis 1988, 1988, 252-254. (b) García Ruano, J. L.; Alemán, J.; Belén Cid, M.; Parra, A. A General Method for the Preparation of N-Sulfonyl Aldimines and Ketimines. Org. Lett. 2005, 7, 179-182.

(11) (a) García Ruano, J. L.; Parra, A.; Yuste, F.; Mastranzo, V. M. Mild and General Method for the Synthesis of Sulfonamides. Synthesis 2008, 2008, 311-319. (b) García Ruano, J. L.; Parra, A.; Marzo, L.; Yuste, F.; Mastranzo, V. M. One-Pot Synthesis of Sulfonamides from Methyl Sulfinates using Ultrasound. Tetrahedron 2011, 67, 29052910.

(12) Harmata, M.; Zheng, P.; Huang, C.; Gomes, M. G.; Ying, W.; Ranyanil, K.-O.; Balan, G.; Calkins, N. L. Expedient Synthesis of Sulfinamides from Sulfonyl Chlorides. J. Org. Chem. 2007, 72, 683685 .

(13) Wei, J.; Sun, Z. tert-Butyl Sulfoxide as a Starting Point for the Synthesis of Sulfinyl Containing Compounds. Org. Lett. 2015, 17, 5396-5399.

(14) Wang, Q.; Tang, X.-Y.; Shi, M. Metal-Free Cross-Coupling of Arylboronic Acids and Derivatives with DAST-Type Reagents for 
Direct Access to Diverse Aromatic Sulfinamides and Sulfonamides. Angew. Chem., Int. Ed. 2016, 55, 10811-10815.

(15) (a) Taniguchi, N. Copper-Catalyzed Oxidative Synthesis of Sulfinamides Using Thiols or Disulfides with Amines. Eur. J. Org. Chem. 2016, 2016, 2157-2162. (b) Dai, Q.; Zhang, J. Direct Synthesis of Sulfinamides by the Copper-Catalyzed Electrophilic Amidation of Sulfenate Anions. Adv. Synth. Catal. 2018, 360, 11231127. (c) Yu, H.; Li, Z.; Bolm, C. Copper-Catalyzed Transsulfinamidation of Sulfinamides as a Key Step in the Preparation of Sulfonamides and Sulfonimidamides. Angew. Chem., Int. Ed. 2018, 57, $15602-15605$.

(16) (a) Nguyen, B.; Emmett, E. J.; Willis, M. C. PalladiumCatalyzed Aminosulfonylation of Aryl Halides. J. Am. Chem. Soc. 2010, 132, 16372-16373. (b) Woolven, H.; González-Rodríguez, C.; Marco, I.; Thompson, A. L.; Willis, M. C. DABCO-Bis(sulfur dioxide), DABSO, as a Convenient Source of Sulfur Dioxide for Organic Synthesis: Utility in Sulfonamide and Sulfamide Preparation. Org. Lett. 2011, 13, 4876-4878.

(17) (a) Emmett, E. J.; Hayter, B. R.; Willis, M. C. PalladiumCatalyzed Three-Component Diaryl Sulfone Synthesis Exploiting the Sulfur Dioxide Surrogate DABSO. Angew. Chem., Int. Ed. 2013, 52, 12679-12683. (b) Deeming, A. S.; Russell, C. J.; Hennessy, A. J.; Willis, M. C. DABSO-Based, Three-Component, One-Pot Sulfone Synthesis. Org. Lett. 2014, 16, 150-153. (c) Rocke, B. N.; Bahnck, K. B.; Herr, M.; Lavergne, S.; Mascitti, V.; Perreault, C.; Polivkova, J.; Shavnya, A. Synthesis of Sulfones from Organozinc Reagents, DABSO, and Alkyl Halides. Org. Lett. 2014, 16, 154-157. (d) Lenstra, D. C.; Vedovato, V.; Ferrer Flegeau, E.; Maydom, J.; Willis, M. C. One-Pot Sulfoxide Synthesis Exploiting a Sulfinyl-Dication Equivalent Generated from a DABSO/Trimethylsilyl Chloride Sequence. Org. Lett. 2016, 18, 2086-2089. (e) Davies, A. T.; Curto, J. M.; Bagley, S. W.; Willis, M. C. One-Pot Palladium-Catalyzed Synthesis of Sulfonyl Fluorides from Aryl Bromides. Chem. Sci. 2017, 8, 1233-1237.

(18) (a) Rosenheim, A.; Singer, L. Die Darstellung von Alkylsulfinsäuren. Ber. Dtsch. Chem. Ges. 1904, 37, 2152-2154. (b) Dragoli, D. R.; Burdett, M. T.; Ellman, J. A. Design, Synthesis, and Utility of a Support-Bound tert-Butanesulfinamide. J. Am. Chem. Soc. 2001, 123, 10127-10128.

(19) (a) Mugford, P. F.; Magloire, V. P.; Kazlauskas, R. J. Unexpected Subtilisin-Catalyzed Hydrolysis of a Sulfinamide Bond in Preference to a Carboxamide Bond in N-Acyl Sulfinamides. J. Am. Chem. Soc. 2005, 127, 6536-6537. (b) Kowalczyk, R.; Edmunds, A. J. F.; Hall, R. G.; Bolm, C. Synthesis of $\mathrm{CF}_{3}$-Substituted Sulfoximines from Sulfonimidoyl Fluorides. Org. Lett. 2011, 13, 768-771. (c) Funes Maldonado, M.; Sehgelmeble, F.; Bjarnemark, F.; Svensson, M.; Åhman, J.; Arvidsson, P. I. Synthesis and Arylation of Unprotected Sulfonimidamides. Tetrahedron 2012, 68, 7456-7462.

(20) (a) Xue, F.; Wang, F.; Liu, J.; Di, J.; Liao, Q.; Lu, H.; Zhu, M.; He, L.; He, H.; Zhang, D.; Song, H.; Liu, X.-Y.; Qin, Y. A Desulfurative Strategy for the Generation of Alkyl Radicals Enabled by Visible-Light Photoredox Catalysis. Angew. Chem., Int. Ed. 2018, 57, 6667-6671. (b) Thota, N.; Makam, P.; Rajbongshi, K. K.; Nagiah, S.; Abdul, N. S.; Chuturgoon, A. A.; Kaushik, A.; Lamichhane, G.; Somboro, A. M.; Kruger, H. G.; Govender, T.; Naicker, T.; Arvidsson, P. I. N-Trifluoromethylthiolated Sulfonimidamides and Sulfoximines: Anti-microbial, Anti-mycobacterial, and Cytotoxic Activity. ACS Med. Chem. Lett. 2019, 10, 1457-1461.

(21) (a) Pemberton, N.; Graden, H.; Evertsson, E.; Bratt, E.; Lepistö, M.; Johannesson, P.; Svensson, P. H. Synthesis and Functionalization of Cyclic Sulfonimidamides: A Novel Chiral Heterocyclic Carboxylic Acid Bioisostere. ACS Med. Chem. Lett. 2012, 3, 574-578. (b) Jacobsen, E.; Chavda, M. K.; Zikpi, K. M.; Waggoner, S. L.; Passini, D. J.; Wolfe, J. A.; Larson, R.; Beckley, C.; Hamaker, C. G.; Hitchcock, S. R. A Mixed Anhydride Approach to the Preparation of Sulfinate Esters and Allylic Sulfones: Trimethylacetic $p$-Toluenesulfinic Anhydride. Tetrahedron Lett. 2017, 58, 3073-3077.

(22) Davies, T. Q.; Hall, A.; Willis, M. C. One-Pot, ThreeComponent Sulfonimidamide Synthesis Exploiting the Sulfinylamine
Reagent N-Sulfinyltritylamine, TrNSO. Angew. Chem., Int. Ed. 2017, 56, 14937-14941.

(23) Fulton, J. R.; Kamara, L. M.; Morton, S. C.; Rowlands, G. J. The Sulfinyl Moiety in Lewis Base-Promoted Allylations. Tetrahedron 2009, 65, 9134-9141.

(24) Meyer, A. U.; Wimmer, A.; König, B. Visible-Light-Accelerated $\mathrm{C}-\mathrm{H}$ Sulfinylation of Heteroarenes. Angew. Chem., Int. Ed. 2017, 56, 409-412.

(25) Wakayama, M.; Ellman, J. A. Recycling the tert-Butanesulfinyl Group in the Synthesis of Amines Using tert-Butanesulfinamide. J. Org. Chem. 2009, 74, 2646-2650. 